\title{
Gene expression profiling identifies pathways involved in seed maturation of Jatropha curcas
}

Fatemeh Maghuly ${ }^{1 *}$, Tamás Deák² ${ }^{2}$ Klemens Vierlinger ${ }^{3}$, Stephan Pabinger ${ }^{3}$, Hakim Tafer $^{4}$ and Margit Laimer ${ }^{5}$

\begin{abstract}
Background: Jatropha curcas, a tropical shrub, is a promising biofuel crop, which produces seeds with high content of oil and protein. To better understand the maturation process of $J$. curcas seeds and to improve its agronomic performance, a two-step approach was performed in six different maturation stages of seeds: 1) generation of the entire transcriptome of J. curcas seeds using 454-Roche sequencing of a cDNA library, 2) comparison of transcriptional expression levels using a custom Agilent 8x60K oligonucleotide microarray.
\end{abstract}

Results: A total of 793,875 high-quality reads were assembled into 19,382 unique full-length contigs, of which 13,507 could be annotated with Gene Ontology (GO) terms. Microarray data analysis identified 9111 probes (out of 57,842 probes), which were differentially expressed between the six maturation stages. The expression results were validated for 75 selected transcripts based on expression levels, predicted function, pathway, and length.

Result from cluster analyses showed that transcripts associated with fatty acid, flavonoid, and phenylpropanoid biosynthesis were over-represented in the early stages, while those of lipid storage were over-represented in the late stages. Expression analyses of different maturation stages of J. curcas seed showed that most changes in transcript abundance occurred between the two last stages, suggesting that the timing of metabolic pathways during seed maturation in J. curcas occurs in late stages. The co-expression results showed that the hubs (CB5-D, CDR1, TT8, DFR, HVA22) with the highest number of edges, associated with fatty acid and flavonoid biosynthesis, are showing a decrease in their expression during seed maturation. Furthermore, seed development and hormone pathways are significantly well connected.

Conclusion: The obtained results revealed differentially expressed sequences (DESs) regulating important pathways related to seed maturation, which could contribute to the understanding of the complex regulatory network during seed maturation with the focus on lipid, flavonoid and phenylpropanoid biosynthesis. This study provides detailed information on transcriptional changes during $J$. curcas seed maturation and provides a starting point for a genomic survey of seed quality traits. The results highlighted specific genes and processes relevant to the molecular mechanisms involved in Jatropha seed maturation. These data can also be utilized regarding other Euphorbiaceae species.

Keywords: Biofuel, Gene expression, High-throughput quantitative real-time PCR, Metabolic pathways, Microarray, Next generation sequencing

\footnotetext{
* Correspondence: fatemeh.maghuly@boku.ac.at

'Plant Functional Genomics, Department of Biotechnology, BOKU-VIBT,

University of Natural Resources and Life Sciences, Muthgasse 18, 1190

Vienna, Austria

Full list of author information is available at the end of the article
}

(c) The Author(s). 2020 Open Access This article is licensed under a Creative Commons Attribution 4.0 International License, which permits use, sharing, adaptation, distribution and reproduction in any medium or format, as long as you give appropriate credit to the original author(s) and the source, provide a link to the Creative Commons licence, and indicate if changes were made. The images or other third party material in this article are included in the article's Creative Commons licence, unless indicated otherwise in a credit line to the material. If material is not included in the article's Creative Commons licence and your intended use is not permitted by statutory regulation or exceeds the permitted use, you will need to obtain permission directly from the copyright holder. To view a copy of this licence, visit http://creativecommons.org/licenses/by/4.0/ The Creative Commons Public Domain Dedication waiver (http://creativecommons.org/publicdomain/zero/1.0/) applies to the data made available in this article, unless otherwise stated in a credit line to the data. 


\section{Background}

Environmental protection and proper land use are some of the main concerns of mankind. Significant emission levels of carbon dioxide $\left(\mathrm{CO}_{2}\right)$ and other greenhouse gases into the atmosphere as a consequence of burning petroleum products for various human activities and its impact on global climate is quite obvious [1]. Actions to mitigate, reduce the effects of climate change (https://www.apha. org/topics-and-issues/climate-change) offer an excellent opportunity to provide innovative methods in order to control air pollution and greenhouse gas emission. Therefore, fuel derived from organic materials (e.g. biofuel crops) receive more attention in the process of shifting from crude fossil oil to more sustainable resources [2]. However, using food crops as first generation biofuels caused the food prices to increase globally, which culminated in a worldwide food crisis. Therefore, methods of biofuel production had progressed from first to second generation, and this novel approach utilizes only non-food crops. Among the second generation biofuels, J. curcas is a promising arable crop, which is frequently mentioned as the best option for marginal quality soils. This plant can be successfully cultivated on soils with low nutrient levels and low water reserves, even in areas that are considered unsuitable for agricultural production.

J. curcas naturally grows in tropical and subtropical climates [3] between sea level and $1800 \mathrm{~m}$ of altitude, and is well adapted to semi-arid, arid conditions and regions with an annual rainfall ranging between 250 and $3000 \mathrm{~mm}$ [4]. J. curcas is a rapidly growing tree that can be propagated easily, and can be used as a multi-purpose plant for biodiesel supply, medicinal uses, veterinary purposes and livestock feed $[5,6]$. The oil quality obtained from the Jatropha crop is very similar to the values of conventional diesel fuel and can be used without any modification in diesel engines currently in operation [7].

J. curcas seed contains non-edible oils, which are traditionally used for soap production and medicinal uses [8]. In addition, its solvents are used due to its therapeutic characteristics by people suffering from various skin diseases and sensitivity to regular soap [9]. All traits mentioned above make J. curcas one of the best candidate as a profitable biofuel crop species for restoring wastelands and improving employment chances and subsistence in rural areas $[10,11]$. Additionally, the $J$. curcas seed cake, which is a waste by-product of the biodiesel trans-esterification process, can be used for the production of various supplies such as organic fertilizer, high-quality paper, energy pellets, soap, cosmetics, toothpaste, embalming fluid, pipe joint cement and cough medicine [12].

$J$. curcas seed kernels are rich in oil (54-58\%) and protein $(20-28 \%)$ compared to the shell, and several efforts were made to make use of cake or kernel meal that remains after oil extraction [6]. Furthermore, it contains a variety of phenolic, flavonoid and diterpenic compounds showing notable anti-oxidant, anti-microbial, and anti-inflammatory activities [5]. However, its toxins and anti-nutritional compounds render the seed cake and oil unsuitable for use as animal feed and human consumption [13]. Therefore, efforts are required to increase oil yield and composition by improving the ability of the plant to produce favorable fruits/seeds with suitable compounds.

Breeding efforts of this biofuel crop will be accelerated by the in-depth knowledge of seed transcripts of J. curcas for obtaining functional genomics information to discover genes that encode enzymes involved in the biosynthesis of oil and toxin precursors and to describe their relevant metabolic pathways [14-16]. Therefore, it is necessary to establish a reliable method to characterize the temporal shifts in gene expression being in the background of the biochemical and metabolic processes which take place during seed maturation. Furthermore, such data could help to identify, characterize and - if necessary - modify the possible transcripts of interest. In Jatropha, transcriptome studies generated data describing seed development and seed germination from manually pollinated plants, with the emphasis on differentially expressed genes related to lipid biosynthesis and toxic compounds [14-18]. In addition, whole genome sequencing was applied to identify protein-encoding genes, which could help in improving the traits of interest (e.g. oil composition) of J. curcas [19-24]. Considering that Jatropha flowering and fruitbearing are practically continuous, the simultaneous presence of mature and immature fruit on the same plant provides a unique opportunity to collect seeds in different stages of maturation of this open-pollinated plant. Therefore, to obtain an overview of transcripts associated with all the seed maturation stages that are potentially involved in seed maturation under the same environmental condition, we performed the following analyses: 1) generation of the entire transcriptome of six stages of $J$. curcas seed maturation using 454-Roche sequencing from pooled samples; 2) comparison of transcriptome expression in seeds of six stages of seed maturation using custom Agilent $8 \times 60 \mathrm{~K}$ oligonucleotide gene expression microarrays.

\section{Results}

Whole seed transcriptome sequencing

To cover the entire J. curcas seed transcriptome, total RNA was extracted from six stages of seed maturation, and equal amounts of total RNA from each sample were pooled together. From this pool, mRNA was isolated and reverse transcribed into cDNA. Normalized cDNA libraries were generated and sequenced using the GS FLX Titanium. Sequencing of cDNA libraries yielded a total of 793,875 high quality (HQ) reads with an average 
read length of 358 nucleotides and 262,096,927 total number of bases (SRX4559398).

After trimming and cleaning, a total of $603,459 \mathrm{HQ}$ reads were assembled into 19,841 contigs (unique transcripts) containing 13,171,840 bases. Out of them, 48, 978 reads were identified as singletons. The size of contigs ranged from 100 to 4088 bases, with 1035 bases as N50 contig size. All contigs can be accessed at http:// short.boku.ac.at/jatropha_contigs. After removing all contaminant sequences, 19,382 unique contigs have been retained. Assembled contigs over 200 bp have been deposited at DDBJ/EMBL/GenBank under the accession GIKD00000000. The version described in this paper is the first version, GIKD01000000.

In addition, the data were compared with the Jatropha genomic sequences of Kazusa DNA Research Institute (JAT_r4.5, ftp://ftp.kazusa.or.jp/pub/Jatropha/) [25, 26] and Chinese Academy of Sciences (JatCur_1.0, ftp://ftp. ncbi.nih.gov/ genomes/Jatropha_curcas/) [27, 28] (Table S1). In total $84,6 \%$ (16,397 contigs), $3.9 \%$ (753 contigs), $1.8 \%$ (351 contigs) showed sequence similarity to both, only to JAT_r4.5 or only to JatCur_1.0 databases, respectively. However, 9.7\% (1881 contigs) were found additionally in the current study. These transcripts are most probably new genes, non-jatropha or non-plant genes, or possibly sequencing artefacts. To assess the quality of the assembled transcripts, the library was compared to the reference transcriptome of JatCur 1.0 available from NCBI. The seed specific de novo RNA library of this study represented about $44.1 \%$ of the 35 , 788 reference RNA coding sequences with an average blast High Scoring Pairs (HSP) coverage of 93\% (S.D. $16.3 \%)$, suggesting a low amount of potential chimeric contigs.

To further assess the extent of represented transcripts in the seed transcriptome, contigs of core eudicot genes were identified using BUSCO. Of the total 2326 core eudicot gene groups, $547(23.5 \%)$ were found in a complete form in our dataset (528 of which were single copy representations suggesting low redundancy in the library). Two hundred sixty fragmented groups were also identified, while 1519 core genes were missing from our dataset. Selected core transcripts were also subject of a phylogenetic analysis, showing that the transcripts are most closely related to J. curcas, Hevea brasiliensis, Manihot esculenta and Ricinus communis, all belonging to the Euphorbiaceae family (Figure S1).

\section{Functional annotation of whole transcript sequencing data}

All 19,382 unique contigs were analyzed by Blast2GO [29] and aligned using BLASTX [30]. Search in the NCBI non-redundant nucleotide database using an Evalue threshold of 1e-6 identified 14,753 unique contigs.
Approximately 3\% (553 contigs) of the transcripts showed top BLAST hits with uncharacterized/predicted proteins, and $24 \%$ (4629 contigs) had no significant similarity to any sequence in the public dataset. The average length of annotated and unannotated contigs was 800 and $400 \mathrm{bp}$, respectively.

GO terms classification identified 13,507 Jatropha unique transcripts received at least one GO annotation (Table S2). The highest percentage of $\mathrm{GO}$ terms was found in the category BP, containing 2409 GO terms, followed by 1841 in MF and 510 in CC (Table S2, Figure S2). The most abundant GO terms in the BP category were genes involved in oxidation-reduction processes (5.1\%), DNA-templated regulation of transcription (3.1\%) and response to cadmium (2.4\%). Within MF, the largest content of functionally assigned ESTs were related to ATP binding (6.5\%), zinc ion binding (5\%) and DNA binding (3.6\%). In CC, the most representative categories were nucleus (14.2\%), plasma membrane (9.1\%) and chloroplast (7.4\%).

Out of the 13,507 sequences annotated with GO terms, 4313 contigs were assigned with 5593 EC numbers representing 1008 unique enzymes, 799 of which are assigned to one or more KEGG pathways (Table S3). Additionally, of the 19,382 contigs, $37.6 \%$ were also annotated based on homology to sequences in the InterPro database (Table S3).

Moreover, 968 different contigs were identified belonging to 20 transporter classes (Table S3). Of them 223 and 236 contigs belong to the transporter classes $2 \mathrm{~A}$ (porters) and 3A (p-p-bond-hydrolysis- driven transporters), respectively.

Protein domain characteristics for Resistance Gene Analogs (RGAs) have been identified in 85 contigs, with 70 carrying a kinase domain, 35 of which also harbored an additional serine/threonine (Ser-Thr) site. RGAs that contain Ser-Thr domain can phosphorylate serine and threonine residues, which are involved in plant development, signaling and defense [31]. However, some RGAs like the Pto gene from tomato encode only Ser-Thr protein kinase. Four contigs with a nucleotide-binding site (NBS-ARC) domain and eight contigs with a leucinerich repeat (LRR) domain were found. Both domains are abundantly present in plants and have an ATPase activity [32].

In addition, 600 contigs could be identified as Transcription factors (TFs), belonging to 52 different TF classes (Table S3). The most abundant TF families were MYB-related, MYB, bZIP, AP2, ERF and RAV, represented by $66,54,49,49,49$ and 46 contigs, respectively.

Annotated sequences were mapped to KEGG pathways showed 2591 contigs located on 143 pathways (Fig. 1, Table S4). Using the KEGG classifications allowed us to identify that the most highly represented pathways were 


\section{9,557 sequences without Enzyme OR Interpro hit 352 transporter 289 TF}

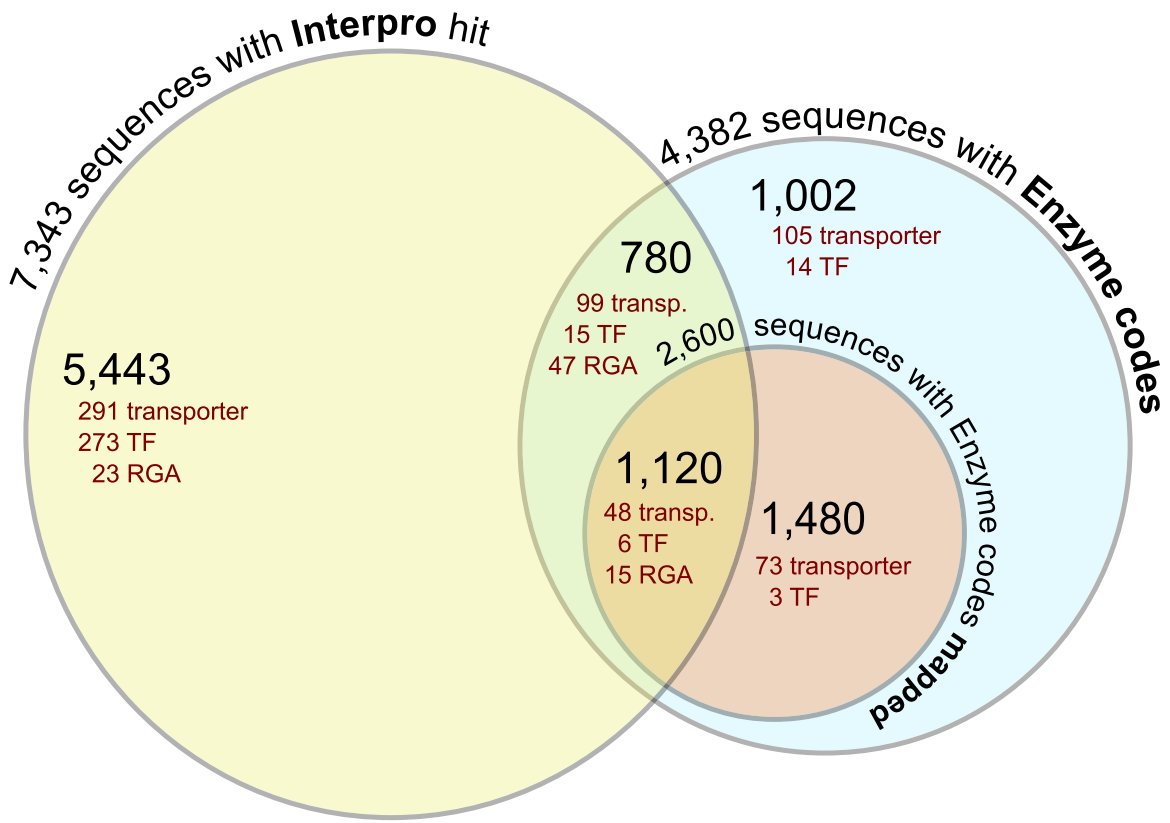

Fig. 1 Venn diagram summarizing the functional annotation process of whole seed transcriptome sequence data. Enzyme codes originate from the Blast2Go annotation, while Interpro hits are resulting from InterProScan (With Interpro hit). Enzyme codes have been checked for mapping on the pathways of the KEGG (With Enzyme codes mapped). Results of the manual annotation for transporters, TFs and RGAs in all classes are also indicated

purine metabolism (315 contigs), followed by starch and sucrose metabolism (226), pyrimidine metabolism (154) and phenylalanine metabolism (138). Further, glycolysis (129), pyruvate metabolism (111), flavonoid biosynthesis (111), glycerolipid metabolism (103) and phenylpropanoid biosynthesis (96) were also found in the top 20 highly represented pathways.

\section{Genome-wide variation in transcript expression during seed maturation}

An 8x60k oligonucleotide microarray containing 57,842 unique probes was produced from 19,841 transcriptome contigs. In total 31,875 specific probes and 2604 crosshybridizing probes (Xhyb) in sense direction, as well as 21,680 specific probes and 1683 Xhyb in antisense direction were designed.

The microarray data were normalized; differential expression patterns were identified, classified, and categorized by their possible molecular function and involvement in metabolic pathways. Principle components analysis (PCA) on transcript expression (abundance) of 57,842 probes showed a clear separation of the six different maturation stages along the first principle component (PC1); which explained 53\% of total variation, and was associated mostly with variation in transcript expression over the maturation stages, where expression from stage IV and V were closer to each other (Figure S3). Significant changes in transcript expression (abundance) were observed in the early and late stages, suggesting a higher physiological differentiation in these stages. In addition, biological replicates of each stage clustered together, suggesting a minimal variation between replicates.

To identify changes in gene expression patterns during seed maturation linear models were calculated and revealed large changes in gene expression over the six stages of seed maturation. A total of 9111 probes (16\% of the total probes) from 7299 contigs $(38 \%$ of the total contigs) were differentially expressed with a $P$-value $<1 \mathrm{e}$ 8 (Table S5 and Figure S4).

The cluster analysis showed that gene different expression patterns could be classified into ten different clusters (1-10) (Fig. 2, 3) of co-expressed genes. Up-regulated transcripts, whose expression was increased during seed maturation, are displayed in clusters 2, 4, 8, 9 and 10 (group A), while down-regulated transcripts were displayed in clusters 1, 3, 5, 6 and 7 (group B).

Besides, we annotated manually the identified differentially expressed sequences (DESs) and patterns with respect to their specific function. Based on the Transporter Classification Database, 431 differentially expressed transcript (699 probes) were assigned to 92 transporter subfamilies, 16 subclasses and 7 classes, distributed among all clusters, representing the intense activities during the maturation process, which requires transport of metabolites within the cell and between different parts of the seed. 

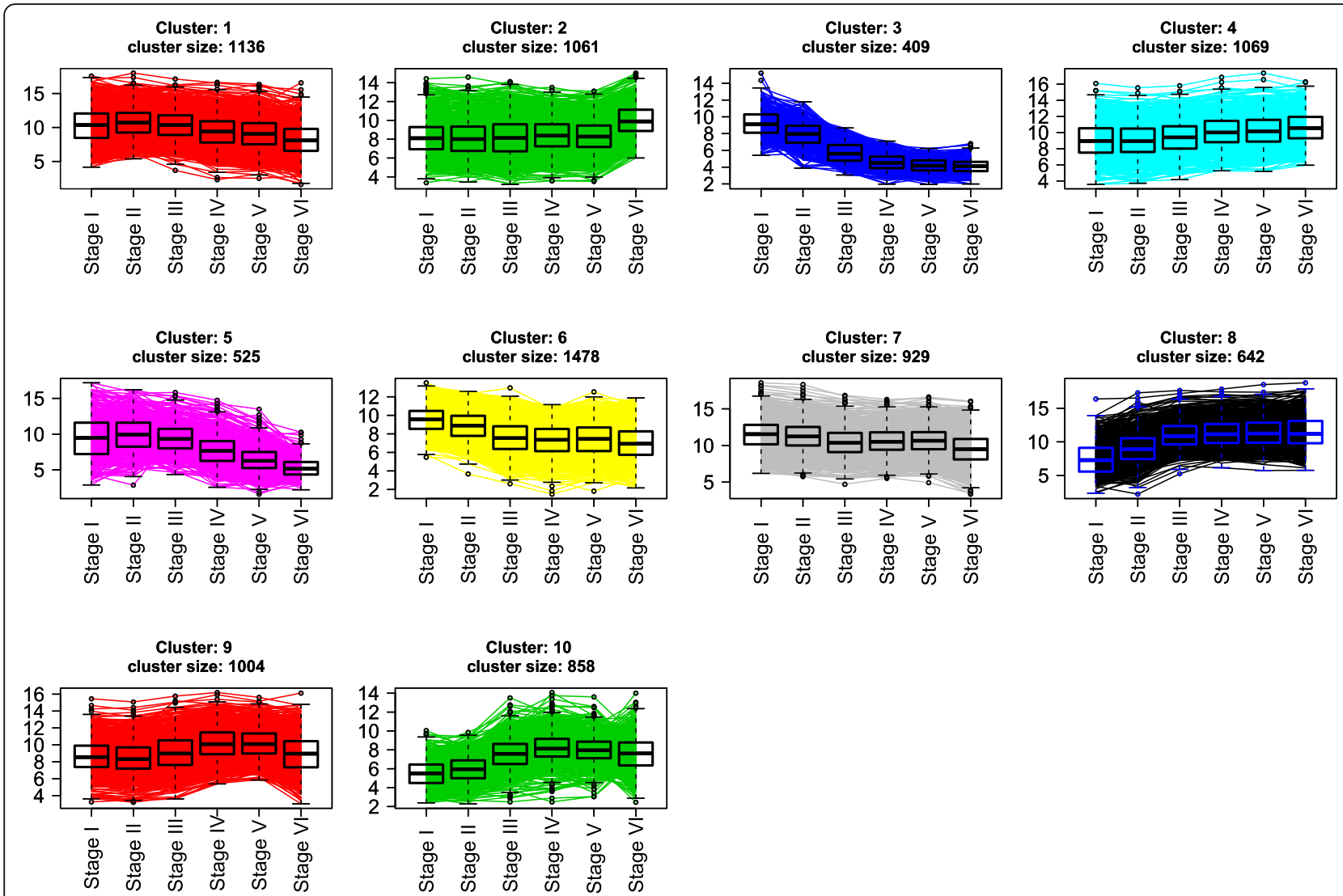

Fig. 2 Cluster analysis of DESs based on their expression patterns across seed maturation stages. The analysis was performed using normalized and filtered data according to their expression profiles between seed maturation stages. Normal mixture modelling for model-based clustering (expectation-maximization) was performed with $P$-value $<1 \mathrm{e}-8$

The highest number (184) of transcripts related to transport activities were identified in class 2 (Electrochemical potential-driven transporters), followed by class 3 (Primary active transporters) with 179 transcripts. Thirteen ranscripts were classified to be transporter subfamily 1.A.33, which is related to heat shock protein (Hsp) 70 (Table S5). Furthermore, different kinds of sugar transporters (2.A.1) and ATP/ADP transmembrane transport (2.A.29) represent the role of transporters to provide necessary energy metabolism during seed maturation. Among 24 transcripts that were classified as $\mathrm{ABC}$ transporters (3.A.1), subfamilies A, C, E, F, G and I were identified (Table S5).

In addition, 47 families of TFs showed differential expression between the six seed maturation stages, involving all expression pattern clusters (Table S5). The highest number of transcripts related to TFs were found in cluster 1, followed by cluster 6 , while the least number of TFs were found in cluster 3 . The most abundant TF families were identified as AP2/ERF-RAV. Furthermore, we explored the co-expression patterns using partial correlation networks. We identified two intermodular hubs with around 40 edges and a broad range of nodes displaying between 15 and 10 edges (Fig. 4a- b). The CB5-D hub showed the highest number of edges followed by unannotated contig02686, CDR1 (aspartic proteinase), unannotated contig00566, TT8 (Transparent Testa 8), unannotated contig19762, and HVA22 (Fig. 4a-b). HVA22), connected CB5-D (cytochrome B5 isoform D), to CDR1, Dihydroflavonol reductase (DFR) and TT8 (Fig. 4a-b).

To focus on processes expected to be involved in seed storage and seed developments as well as hormone pathway, the GO terms from BP category were extracted. The co-expression results showed a high degree of connectivity between seed development and hormone pathways, while seed storage is less connected to the other two pathways (Fig. 4c).

In addition, based on pairwise comparison between seed maturation stages, the highest number of differentially expression transcripts related to 889 and 272 contigs (1122 and 1673 probes), which were found between stages V and VI (Table S6).

\section{GO enrichment analyses of DESs}

GO enrichment analyses in BP categories for each cluster indicated that the most significantly over- and under-represented DESs were found in cluster 6 (Top 15 


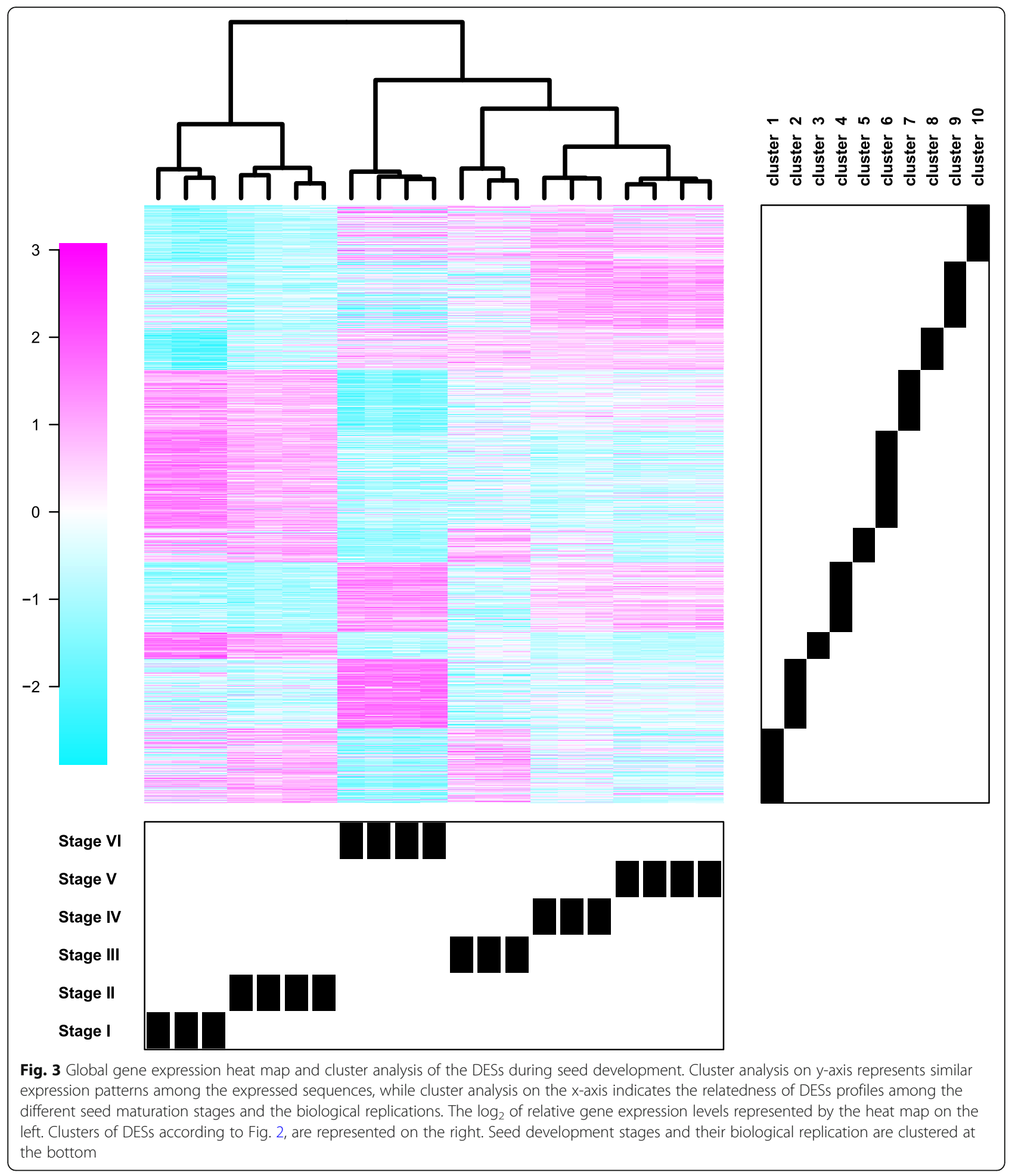

GO terms and detailed information for each cluster are shown in Fig. 5, Figure S5, Table S7 and Table S8).

Besides, visualization of enriched GO terms related to BP category showed that the GOs related to fatty acid metabolism (e.g. unsaturated fatty acid, linoleic acid), lipid storage, dormancy process, aromatic acid transports, monoterpenoid metabolism, and UDPglucose metabolism were significantly over-represented in cluster group A, with higher DES level during late stage of seed maturation. Furthermore, Raffinose family oligosaccharides (RFOs), which are associated with late maturation in Arabidopsis, Brassica napus and Medicago 


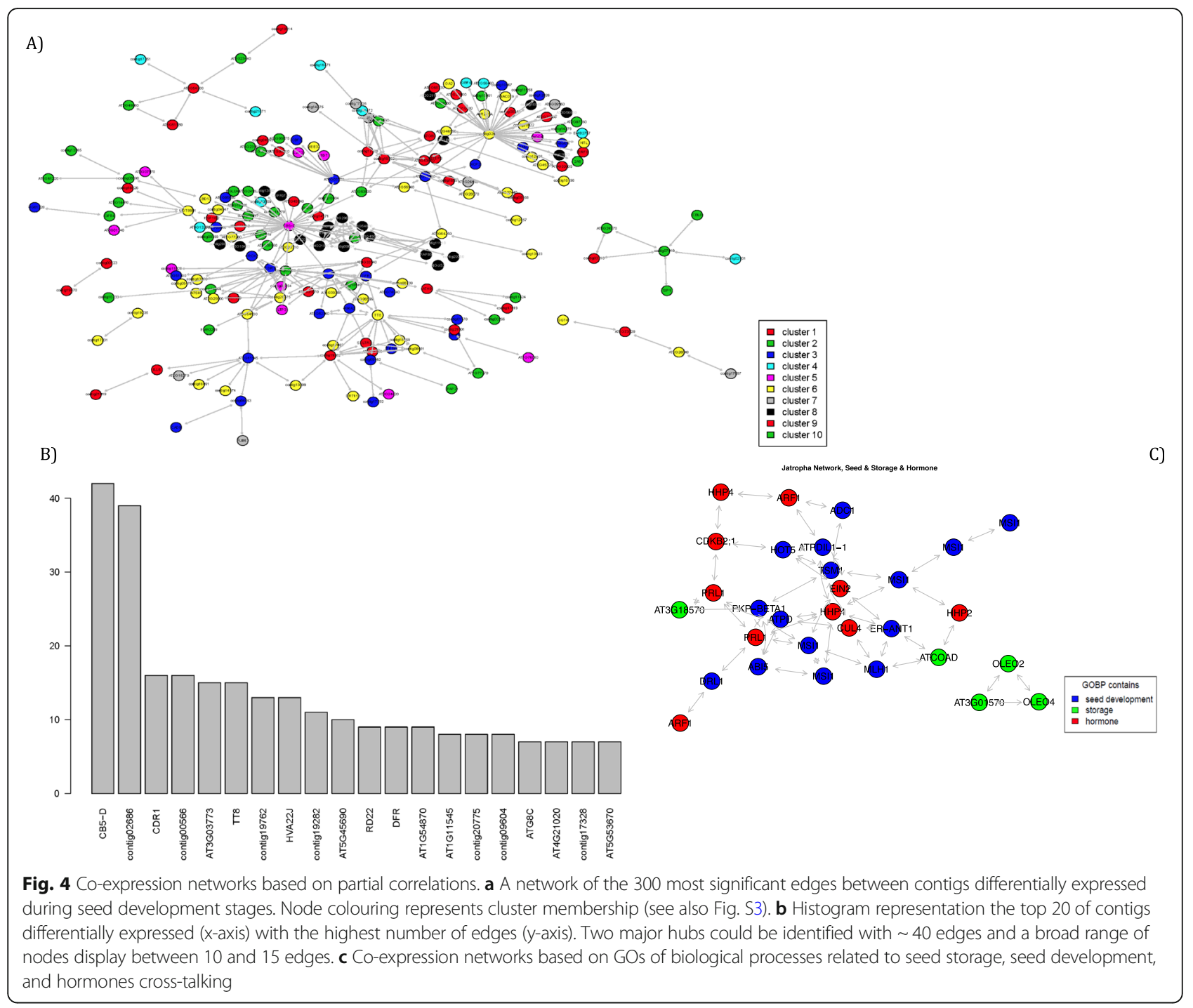

trunculata [33-35] and transcripts related to biosynthesis of serine and glycine, Embryo sac development, RNA modification, methylation, maintenance of seed dormancy, protein folding and RNA modification were over-represented in this group.

In contrast, GOs related to phenylpropanoid and flavonoid metabolism and biosynthesis, as well as cell wall modification and carbohydrate metabolism in cluster group B, were significantly enriched with high expression levels during the early stage of seed maturation (Figure S4). Transcripts involved in hormone transporters, signaling, ATP hydrolysis coupled protein transport and purine ribonuclease metabolism were significantly overrepresented in this group.

GOs involved in glucan and beta-glucan biosynthesis playing a key role in regulating seed coat-imposed dormancy [36] were over-represented in two clusters (3 and 8 , respectively). Also, GOs involved in translation, RNA processing, and gene expression were under-represented in different clusters (6 and 9). They represent a different pattern of gene expression during seed maturation, indicating the involvement of two different groups of genes.

\section{KEGG enrichment analysis of DESs}

To further understand the biological function of DESs during seed maturation, enriched KEGG pathways with $P$-value $<0.05$ in the set of DESs was assessed (Table S8 and S9, Figure S4 and S6).

\section{Pathway enrichment in DESs related to lipid metabolism}

In the plant, pathways contributing to lipid biosynthesis can be divided into three steps and cell compartments; a) fatty acid biosynthesis in the plastids, b) triacylglycerol (TAG) biosynthesis in the endoplasmic reticulum (ER), and c) oil body formation in the cytoplasm [37]. Altogether, 97 contigs and 55 enzymes distributed 


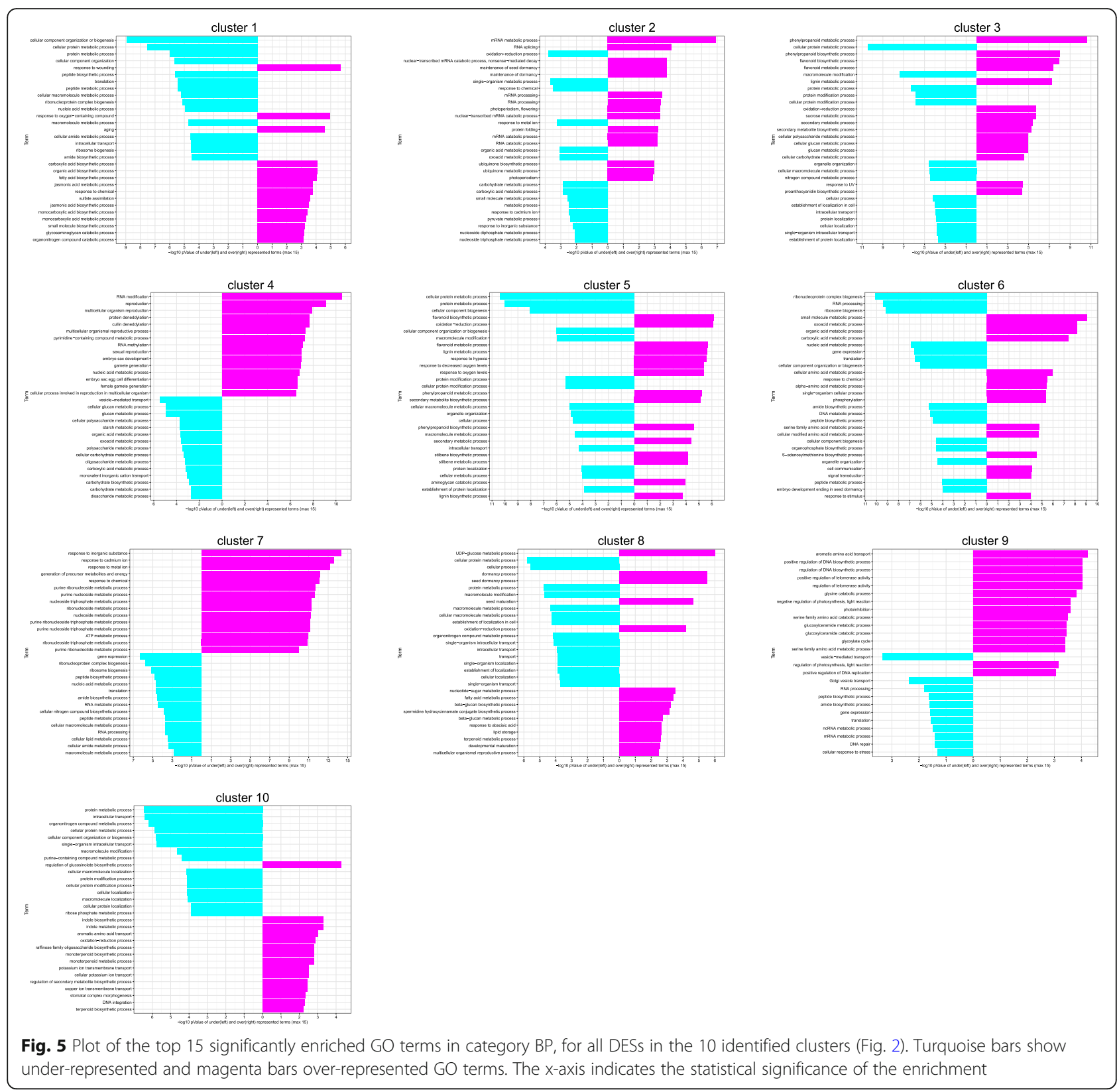

among 13 pathways were enriched as being involved in these steps (Figure S7).

In the first step, we could identify DESs related to 3oxoacyl-ACP reductase (KAR, EC:1.1.1.100), containing various DESs (five contigs in clusters 1 and 8). In addition, Beta-ketoacyl-ACP synthase I (KASI, EC: 2.3.1.41, two contigs in clusters 5 and 8) with DESs were identified showing different regulation patterns. The elongation from $16: 0$-ACP or $18: 1$-ACP [38] occurs in the plastid and is catalyzed by acyl-ACP desaturase (AAD, EC:1.14.19.2), which was represented in current study by four DESs (in clusters 5 and 8). Additionally, we identified Oleoyl-ACP hydrolase (OAH, EC:3.1.2.14, two contigs in clusters 1 and 5), removing acyl group from ACP, and Acyl-CoA synthetase (EC:6.2.1.3, two contigs in cluster 8 ) engaged in glycerophospholipid metabolism and fatty acid elongation.

We also identified key enzymes involved in triacylglycerol (TAG) production such as phospholipid: diacylglycerol acyltransferase (PDAT1, EC:2.3.1.158, two contigs in cluster 8), lysophosphatidic acid acyltransferase (LPAAT, EC:2.3.1.51, five contigs in clusters 2 and 4), and PA phosphatase (PAP, EC:3.1.3.4, one contig in cluster 2). Two DESs in cluster 10, encoding diacylglycerol O-acyltransferase (DGAT, EC: 2.3.1.20), and two DESs corresponding phospholipid diacylglycerol acyltransferase (PDAT, EC:2.3.1.158) in cluster 8 
were identified. Furthermore, triacylglycerol lipase (EC: 3.1.1.3, six contigs in clusters 4 and 8), which modifies TGA into fatty acids was identified. All DESs involved in TAG production process were classified in clusters 2,4 and 8 , showing their expressions increase during seed maturation.

Contigs encoding enzymes like diacylglycerol kinase (ATP, EC:2.7.1.107, two contigs in cluster 4), aldehyde dehydrogenase (NAD+, EC:1.2.1.3, three contigs in clusters 2, 4 and 8) and glycerate 3-kinase (EC:2.7.1.31, two contigs in cluster 2), were found in cluster 2,4 and 8 , showing an increase during the last stage of $J$. curcas seed maturation. As expected, the synthesis of fatty acids requires a high amount of energy during seed maturation, which results in increased expression of enzymes related to photosynthesis as an energy supply [39].

Furthermore, two important enzymes involved in alphalinolenic acid metabolism and biosynthesis of unsaturated fatty acids were identified in cluster 1: acyl-CoA oxidase (EC: 1.3.3.6, one contig) and enoyl-CoA hydratase/3-hydroxyacylCoA dehydrogenase (EC:4.2.1.17, two contigs).

\section{Pathway enrichment in DESs related to phenylpropanoid biosynthesis}

Among the significantly enriched pathways, the phenylpropanoid biosynthesis pathway contained 30 overrepresented contigs and 11 enzymes located in clusters 3, 5 and 8 (Figure S8). In this pathway, we identified two over-expressed transcripts (contig05064, and contig05269) related to phenylalanine/tyrosine ammonialyase (PTAL, EC:4.3.1.25), the expression of which decreases during seed maturation as shown in clusters 3 and 5. Furthermore, one transcript corresponding to trans-cinnamate 4-monooxygenase $(\mathrm{C} 4 \mathrm{H}, \mathrm{EC}: 1.14 .13 .11$, one contig in cluster 5) was identified, which converts cinnamic acid to P-coumaric acid. Finally, P-coumaric acid can be conjugated by 4-coumarate: CoA ligase (4CL, EC: 6.2.1.12) and enriched to coenzyme A to form p-coumaroyl-CoA, which is the precursor for the synthesis of flavonoids, stilbenes, and other phenylpropanoids [40]. For this enzyme we also identified five transcripts, enriched in clusters 3 and 5 .

Caffeoyl-CoA O-methyltransferase (CCoA-OMT; EC: 2.1.1.104) with four transcripts was over-represented in clusters 3,5 , and 8 . Finally, 11 transcripts in clusters 3 and 5 for peroxidase (EC:1.11.1.7) were significantly enriched and over-represented.

\section{Pathway enrichment in DESs of flavonoid biosynthesis- related pathways}

After oil extraction, the Jatropha seed cake contains high amounts of polyphenols and pigments as a result of flavonoid biosynthesis. In this study, $42 \mathrm{DESs}$ were annotated and enriched in clusters 1, 3 and 5. They encoded 16 enzymes involved in flavonoid, flavone and flavonol biosynthesis and isoflavonoid biosynthesis (Figure S9). Two differentially expressed transcripts (cluster 1 ) were identified as 6'-deoxychalcone synthase (EC:2.3.1.170) and three transcripts (clusters 3 and 5) as naringeninchalcone synthase (CHS, EC:2.3.1.74), an important enzyme catalyzing the conversion of cinnamoyl-CoA to pinocembrin chalcone. One transcript was annotated for chalcone isomers (CHI, EC:5.5.1.6, cluster 3) that catalyzes the conversion of pinocembrin chalcone to pinocembrin, a substrate for galangin synthesis [41]. Four transcripts were identified as flavanone 3-dioxygenase or naringenin 3-dioxygenase (F3H, EC:1.14.11.9, in cluster $5)$, which is involved in highly conserved pathways in plants to convert naringenin into dihydrokaempferol. It is an important intermediate product, that can be converted to kaempferol by flavonol synthase (EC: 1.14.11.23), identified in the current study with 10 differentially expressed transcripts (in clusters 1, 3 and 5). The presence of different expression patterns (from different clusters) of one enzyme could be explained by the existence of different isoenzymes and possibly by the interaction with other genes involved in flavonoid biosynthesis at multiple loci [42].

\section{Validation of microarray data using qRT-PCR}

A total of 70 contigs from the DESs represented transcripts in seeds, and three housekeeping genes were selected (Table S10 and Table S11) and used for independent validation using a 48.48 chip (Fluidigm) to confirm that the changes in expressions as indicated by microarray data were authentic and reliable. Candidates for qPCR were chosen based on expression levels, known function, clusters and length of contigs. Additionally, some contigs of unknown function were selected. The corresponding primers are listed in Table S10.

The expression patterns obtained by qRT-PCR correlate strongly to moderately with data from the microarray analyses (about half of the contigs correlate with the microarray data at a Pearson correlation $<-0.8$ ), thus confirming the reliability of the chosen approach (Figure S10).

\section{Discussion}

The understanding of transcriptional variation during different seed maturation stages is of utmost importance for breeding strategies in J. curcas; especially for low anti-nutritional, high-quality oil, and bioactive component levels, which could make the crop suitable for biodiesel, animal feed and pharmaceutical use. In this study, genome-wide transcriptome analysis was used to identify the global gene expression pattern of J. curcas seeds at six different developmental stages, collected at the same time point on one plant. Even though J. curcas is an important oil crop, this is the first study of profiling 
genome-wide transcript expression during seed maturation of an open-pollinated plant.

The sequencing of the whole seed transcriptome of J. curcas revealed 19,382 unique contigs, of which 14,753 contigs were aligned through Blast search; however, 4,629 contigs could not be annotated since they did not have any BLAST hits. The high number of unannotated transcripts might be an indication of potential limitations in transcriptome assembly and annotation. The unannotated sequences could include both novel transcripts and technical artifacts from the sequencing technology (library preparation and/or sequencing machine). Additionally, the applied BLAST parameters are optimized for complex full-length RNA sequences, which does not favor BLAST searches of short (150-200 bp) sequences of low complexity. Furthermore, the comparison of contigs with different Jatropha genome sequences [19-22] revealed additional 1881 contigs in the transcript data set of the current study.

On the other hand, use of whole seed transcripts of different maturation stages allows us to design a robust and high coverage microarray platform (in J. curcas) to compare a constant large number of genes for the expression evaluation of different genotypes, organs, and tissues. Although RNA-Seq has some superior benefits for quantitative transcriptomics, microarray is still a common method of choice, since in compared to RNA-seq, microarray is cost effective, fast and provides concordant results. Besides, bioinformatics and statistics practices for microarrays are well established and straightforward in comparison to RNA-seq data, which is more complex [43].

The examination of genome-wide variation in transcripts at selected seed developmental points could allow us to identify DESs with the high number of edges that might be associated with lipid and flavonoid biosynthesis as well as unknown functions. It was also noteworthy to find that all DESs with high number of connectivity belong to cluster group B showing a decrease in their expression during seed maturation. The co-expression patterns showed that the CB5-D hub has the highest number of edges, followed by unannotated contig02686 (Fig. 4a-b). The CB5, a cytochrome B5 isoforms, and small tail-anchored membrane proteins play an essential role in many cellular processes, including lipid biosynthesis. It is well known that CB5-D in different morphotypes of Brassica rapa provide electrons for various enzymes located in the endoplasmic reticulum (ER), including fatty acid desaturase (FAD), FAD-like proteins, and are also physiologically important for $\mathrm{p} 450$ protein family [44-48]. Hwang et al. [45], combined in vivo and in vitro assays to show that CB5-D are targeted exclusively to mitochondrial outer membranes, while the other isoforms of CB5 (A, B, and C) are targeted to the ER. In addition, our result showed a direct connection between CB5-D from cluster 5 and CDR1 from cluster 3
(Fig. 4a). Although proteins with hydrolase activity like CDR1 do not imply the production of seed oil, overexpression of microsomal DGAT1 - a key enzyme of triacylglycerol production - resulted in differential regulation of CDR1 expression in transgenic and untransformed control in Brassica [49]. Furthermore, CDR1 and CB5$\mathrm{D}$, show the highest expression in the early stage of seed maturation, suggesting their indirect roles in fatty acid biosynthesis in the early stages.

Besides, the contig02686 (unannotated) from cluster 6 also contains a high number of edges, which may be connected to some hypothetical proteins. There is a need for functional analysis of this contig, which might support important biological cell functions and could potentially serve as targets for further studies.

Along with the hub with the high number of edges, TT8 from cluster 6 has an essential role in the regulation of flavonoid biosynthesis and the formation of seed coat colour. However, Chen et al. [50] reported that TT8 also affect FA biosynthesis in seeds of Arabidopsis maternally, which also inhibits FA accumulation by down-regulating of the expression of a carboxylase biotin carboxylase subunit (CAC2), beta-ketoacyl-acp synthetase II (KASII), mosaic death1 (MOD1), fatty acid biosynthesis2 (FAB2), acyl-acp thioesterase (FatA), fatty acid elongation1 (FAE1), FAD2 and FAD3, all playing an important role in FA biosynthesis during seed maturation. The TT8 also represses the expression of leafy cotyledon 1 (LEC1), LEC2, FUSCA3 (FUS3), and cytidine diphosphate diacylglycerol synthase2 (CDS2), which are critical to embryonic development [50]. On the other hand, TT8 influences DFR expression, which commits phenolics to proanthocyanidins synthesis responsible for seed coat and quality germplasm of canola [51]. In Arabidopsis, At4g09820 (TT8) encodes a protein, which is important in the expression of DFR, while DFR can give rise to flavonoids [52], representing their important role in flavonoid pathways. The high connectivity and similar expression patterns on both annotated transcripts in the current study might suggest the same functions and roles in J. curcas.

Eventually, HVA22-like protein - a stress-inducible gene [53] from cluster 3 , playing a role as a hub connection among DFR, TT8, and CDR1, represents its effect on fatty acid and flavonoid biosynthesis pathways in $J$. curcas. HVA22 is identified to be an ER- and Golgilocalized protein and is able to regulate gibberellinmediated vacuolation negatively [53].

Considering the intense metabolic activity during seed maturation, which requires the regulation of target genes and the exchange of metabolites and proteins between different locations in seed and within the cell, it is important to identify transcripts related to transport machinery and TF. Among the differentially expressed transcripts that were classified as a transporter, 
subfamily 1.A.33 were related to Hsps, which perform diverse biological functions in collaboration with chaperons either in stress or non-stress conditions. In the absence of heat stress, $H s p$ genes are accumulated during the late stage of seed maturation [54]. Several plant cytosolic Hsp70 were identified during development, maturation, and germination of seeds of pea and Arabidopsis $[55,56]$. In this study, three transcripts were identified as homologues to Arabidopsis BiP1 of plant Hsp70 family. These genes appeared to be highly expressed in the early stage of seed maturation (cluster 6), which is in concordance with previous results, where BiPs (BiP-1 and $B i P-2)$ showed higher expression during the early stage of seed development, which decreased toward the end of seed maturation. These are related to the BiP roles in rapid cell expansion, accumulation of seed storage protein, and seed maturation [56-58].

In the group of $\mathrm{ABC}$ transporters (3.A.1) which are involved in plant development, nutrition, stress response and phytohormones and primary metabolites transports [59], one transcript showed homology to AtABCG14 (cluster 5 and 10), described to be involved in translocation of cytokinins between the root and the shoots in Arabidopsis [60, 61]. This transcript could be essential for long-distance communication between root-shootfruit as well. It was also reported that the $\operatorname{tg} d 1$ (trigalactosyldiacylglycerol) mutants showed a decrease in ERderived plastid lipids, and accumulation of oligogalactoglycerolipids (TGDG) and TAG in leaf tissues [62], showing that TGD1/ AtABCI14 encodes a membranespanning protein [59].

We also identified one ABCG reporter transcript in cluster 10 homologous to Arabidopsis AtABCG25, reported to act as a carrier to export ABA from the vascular tissues, where it is mainly produced [63]. In addition, one homolog of AtABCD1 found in cluster 4 and cluster 9 facilitated the transport of lipidic metabolites in Arabidopsis [64]. Moreover, transcripts associated with transporters AtABCC2 and AtABCI17 expressed in cluster 7 and 9 , respectively, were reported to be involved in transport of toxic compounds in Arabidopsis. AtABCC2 is tolerant to metals and act as chlorophyll catabolic transporter, while AtABCI17 is expressed in roots and is highly sensitive to Aluminium [59]. It is clear that plant $\mathrm{ABC}$ transporters play an important role for survival of plant and seed maturation; however, many questions remain to be answered since only a few of the plant $A B C$ transporters were functionally analyzed (22 out of 130 in Arabidopsis) [59, 64].

Transcripts related to TFs are distributed among all clusters with different expression patterns. Among TFs related to seed oil accumulation, we could identify differentially expressed transcripts that showed homology to Arabidopsis AP2 type TFs, WRI1 and HD-ZIP type,
GLABRA2 (Table S5). The WRI1 has been studied extensively in the regulation of the transcription levels of genes in lipid biosynthesis pathways in the Arabidopsis, rapeseed, maize, potato, Siberian apricot kernel, and Jatropha seeds [24, 65-69]. It was reported that overexpression of JcWRI1 not only increased the lipid content but also the seeds mass. In addition, overexpressing JcWRI1 in Jatropha seeds increased oleic acid (C18:1) level compared to linoleic acid (C18:2), which also increased the expression level of enzymes related to oleic acid production such as BCCP2, KASI, KASIII, FATA, ACP1, and DGAT1 [24]. In the current study, WRI1 and KASI of cluster 5 presented similar expression patterns and were highly expressed in the early stage of seed maturation. This could indicate that the function of both genes closely correlates with fatty acid biosynthesis in the early stage of seed maturation. This is also in agreement with the previous report in Arabidopsis which found that WRI1 targeted many genes from FA synthesis [70].

Furthermore, GLABRA2 (GL2), a member of the HDZIP family, identified in cluster 3, exhibited a downregulated expression pattern during seed development, which is in contrast with the expression pattern of identified enzymes related to lipid biosynthesis pathway, where their expression increased with development of the seed. Based on previous studies, GL2 was a negative regulator of seed oil content [71], which is in agreement with the current data.

Since the major goal of seed oil crop research is focused on oil quality and quantity, it is necessary to understand the processes involved in seed metabolism [5]. On the other hand, phenolic compounds, which are produced under optimal and suboptimal conditions, could influence and improve seed development, germination, metabolism, and biomass accumulation [72]. Furthermore, Synthesis of phenols involved in several pathways, such as flavonoid and phenylpropanoid biosynthesis pathways, could help the plant to cope with different stress conditions [73]. Therefore, focusing on DESs related to enzymes involved in lipid, flavonoid, and phenylpropanoid biosynthesis pathways is of great interest.

In the current study, most of the enzymes involved in lipid biosynthesis in J. curcas were identified based on the annotation of the seed transcripts. Among key enzymes involved in fatty acid concentration in the plastid, KASI was identified with two quite different expression patterns during seed maturation stages, suggesting their functional differentiation during seed maturation. However, Jiang et al. [15] and Xu et al. [74] found that the expression of the KASI gene increased during seed maturation in J. curcas. On the other hand, among key enzymes involved in TAG synthesis, LPAT, DGAT, and PDAT were identified in our dataset, showing that they 
were upregulated during seed maturation, indicating their essential roles in the synthesis of TAG. These genes were also represented by a different number of transcripts, expression level, and pattern, showing that they contain various isoforms with different functions.

A previous study reported five genes for the LPAT in the Arabidopsis genome and demonstrated different expression patterns and functions. Three of them (LPAT1, LPAT2, and LPAT3) are essential to normal plant development, where over-expression of LPAT2 improved accumulation of TAG in seeds [75].

It is also well known that DGAT, a key enzyme to the synthesis of TAG, could improve the oil content in Arabidopsis, Brassica napus, soybean, and maize seeds [76, 77], and therefore, this step has received the most attention to increasing amount of TAG [77].

In plants, DGATs encode by two genes (DGAT1 and $D G A T 2)$, which were also identified in Jatropha $[5,14]$. However, genetic studies with mutants showed that the disruption of the DGAT1 gene reduces $70-80 \%$ of oil content in Arabidopsis seeds. Besides, the function of DGAT2 is unclear in the Arabidopsis mutant [77, 78]. The expression patterns of the two DGAT were also studied in developing seeds of soybean, Euphorbia, castor bean, and Jatropha [74, 79]. It was reported that in Jatropha, DGAT2 mainly expressed in leaf and poorly expressed in developing seeds. In contrast, the castor bean showed the expression of DGAT2 at a higher level compared to DGAT1 in developing seeds [77].

PDAT has various isoforms, but PDAT1 showed to have an important role in seed TAG content in Arabidopsis [80]. Using various RNAi silencing of PDAT1 and DGAT1 showed that both genes have an overlapping role in the synthesis of TAG in oil-seed plants. However, their expression was reported to be different in various plants. For instance, in sesame PDAT showed higher expression compared to DGAT, while in castor bean the DGAT showed a higher expression level compared to PDAT [14, 81, 82]. In Jatropha, PDAT showed lower expression compare to DGAT1, as reported by $\mathrm{Xu}$ et al. [74]. However, Ha et al. [14] described a higher expression of PDAT compared to DGAT. In the current study, PDAT and DGAT showed the highest expression in the last stage of seed maturation. However, the expression of PDAT starts to increase from the middle stages of seed maturation, while considering DGAT, the expression starts to increase in the late stages (Table S5, Figure S4), indicating that DGAT has more important role in later stages compared to PDAT.

The transcripts involved in the expression of the triacylglycerol and FA desaturation biosynthesis processes increased during middle to late stages of seed maturation, something previously reported in Brassica rapa [83], which is also in agreement with the current results. This could be explained by the rise of storage lipid production, which is also confirmed by previous research studies $[17,18,84-86]$.

In this study, we also identified the expression pattern of various key enzymes involved in phenolic compounds during seed maturation of $J$. curcas. It is noteworthy to point out that, in many cases, they are present in multiple copies with different expression patterns. For instance, previous reports showed that 4CL genes contain four isoforms which differ in terms of localization and activity in Arabidopsis. In Arabidopsis, the 4CL3 has shown to be expressed in a broad range of cell types, and is mainly co-expressed with flavonoid biosynthesis pathways, while 4CL1, 4CL2, and 4CL4 are associated with lignin biosynthesis genes [79, 87, 88]. The results are in agreement with our data, where $4 \mathrm{CL}$ were identified with 5 DESs.

The flavonoid biosynthesis pathway is well conserved among plants [89]. Considering that the expression level of most transcripts related to flavonoid biosynthesis was more than two times down-regulated in the last stage compared to early stages in all three clusters $(1,3,5)$ (Fig. 2), it is suggested that the genes involved in flavonoid biosynthesis may be essential during the early stages of seed development [42]. Six DEGs involved in flavonoid biosynthesis pathways were significantly enriched in male and female flower buds of J. curcas, and all of them were up-regulated in male vs. female flowers. The expression pattern of major flavonoid biosynthesis genes was also down-regulated during seed development in Arabidopsis thaliana [90]. However, the expression of each of these contigs did not follow a similar pattern during later stages, and even three contigs of the enzyme flavonoid 3', 5' -hydroxylase (EC: 1.14.13.88), present in two different clusters ( 1 and 3 ) that may indicate the presence of different isoenzymes and functions. Therefore, since flavonoids are involved in protective function [91], it is important to understand the functional role of these transcripts.

\section{Conclusions}

Different approaches were used to identify sets of genes with various transcript abundance during seed maturation. First, to obtain an overview of the variation in seed maturation stages, PCA was carried out using all transcripts present in the microarray (Fig. S3). The first principle component (PC1: 53\% explained variation) analysis captured mostly temporal variation in transcript abundance, which is supported by previous studies in Brassica rapa [83], and Arabidopsis thaliana [92, 93], where seed developmental stages are the major source of transcriptional and metabolic variation.

Second, the center of our attention was directed to transcripts related to Jatropha seed maturation to 
correlate co-expression patterns within pathways and to anticipate putative regulatory elements of the metabolisms of interest (Fig. 4). The co-expression analysis showed that the CB5-D had the highest number of edges, connected directly to CDR1. The co-expression result showed a high degree of connectivity between seed development and hormone pathways, while seed storage is less well connected to the other two pathways.

Third, a subset of probes with variation in transcript abundance patterns between maturation was selected for further analyses. This subset of genes was present in different clusters, which were enriched in various metabolic pathways such as fatty acid biosynthesis, flavonoid biosynthesis, glucan metabolic biosynthesis, seed maturation and dormancy, sucrose and hormone metabolic processes.

Fourth, pairwise transcript expression analyses of different maturation stages of $J$. curcas seeds showed that most changes in transcript abundance occurred between stages V and VI with brown and black epicarp, respectively (Table S6), suggesting that the timing of metabolic pathways during seed maturation in J. curcas is in late stages. The expression results were validated for $75 \mathrm{pu}$ tative transcripts.

Finally, cluster analyses were used to discover particular seed maturation-dependent patterns of gene expression. Transcripts related to fatty acid, flavonoid, and phenylpropanoid biosynthesis were over-represented in the early stage, while lipid storage in the late stage. Generally, the expression of the most over-represented transcripts decreases in the last stage of seed maturation.

\section{Methods}

\section{Plant material}

Seeds of a selected J. curcas plant in Kamisse, Ethiopia, were collected at six maturation stages (I-VI) and characterized according to the color of epicarp and endocarp [green-white (I), green-brown (II), green-black (III), yellow-black (IV), brown-black (V), dry-black (VI)] [94] at the same time. Three biological replicates were used for each sample, immediately frozen in liquid nitrogen and stored at $-80^{\circ} \mathrm{C}$.

\section{Total RNA extraction}

Total RNA was extracted from six stages of seed maturation of $J$. curcas using plant RNA purification reagents (Invitrogen) according to the supplier's instructions. The quality and concentration of total RNAs were determined using NanoVue Spectrophotometer (GE Healthcare Life Sciences) and gel electrophoresis. All RNA samples showing A260/280 ratios between 2.0 and 2.15 were selected and analyzed for RNA integrity using an Agilent 2100 Bioanalyzer (Agilent Technologies). RNA samples with an integrity number above 7.0 were used for further analyses.

\section{cDNA synthesis for sequencing}

Equal amounts of extracted RNA from different seed maturation stages were pooled and used for cDNA library construction. To purify mRNA from $5 \mu \mathrm{g}$ total RNA, the mRNA-Only Eukaryotic mRNA Isolation Kit (Epicentre) was used by applying exonuclease digestion followed by $\mathrm{LiCl}$ precipitation. One $\mu \mathrm{g}$ mRNA was used for the synthesis of the first-strand cDNA by the Mint-Universal cDNA Synthesis Kit (Evrogen). The Trimmer Kit (Evrogen) was used for normalization reaction using $800 \mathrm{ng}$ amplified cDNA, which was re-amplified by 18 cycles.

\section{Size selection and cloning of cDNA}

Two $\mu \mathrm{g}$ of normalized cDNA were digested by ten units of the SfiI restriction enzyme (New England Biolabs) for $2 \mathrm{~h}$ at $48^{\circ} \mathrm{C}$. Fragments (> $800 \mathrm{bp}$ ) isolated from an LMP agarose gel were purified using the MinElute Gel Extraction Kit (Qiagen). The Fast Ligation Kit (New England Biolabs) was used for ligation of $200 \mathrm{ng}$ purified cDNA fragments to 100 ng SfiI using dephosphorylated pDNR-lib Vector (Clontech). The product was desalted by ethanol precipitation and redissolved in $10 \mu \mathrm{l}$ water. Of this, $1.5 \mu \mathrm{l}$ was used to transform NEB10b competent cells (New England Biolabs). To verify the success of normalization, 96 clones were randomly selected and sequenced.

\section{cDNA library preparation and sequencing using Roche 454 FLX}

One million clones were plated on LB-Cm agar plates, collected and stored in glycerol stocks at $-70{ }^{\circ} \mathrm{C}$. Half of the cells were inoculated to a $300 \mathrm{ml}$ Terrific Broth/ $\mathrm{Cm}$ culture and were grown for $5 \mathrm{~h}$ at $30^{\circ} \mathrm{C}$. One hundred Units Sfil digested $200 \mu \mathrm{g}$ of purified plasmid DNA (Qiagen) for $2 \mathrm{~h}$ at $48^{\circ} \mathrm{C}$. LMP-Agarose/MinElute Gel Extraction Kit was used to purify inserts, which were ligated to high-molecular-weight DNA using a Sfilinker.

The library for the Roche 454 FLX sequencing was generated according to the manufacturer's protocols (Roche/454 Life Sciences). The concatenated inserts were sheared to fragments ranging from 400 to $900 \mathrm{bp}$. The two 454 A and B adaptors were ligated to the ends of the emulsion PCR and sequencing. The library was sequenced on one picotiter-plate of the GS FLX using the Roche/454 Titanium chemistry.

\section{Assembly of the sequence reads to transcripts}

At first, the reads were screened for the Sfi-linker used for concatenation and linker sequences were removed. The Roche/454 Newbler software (454 Life Sciences Corporation, Software Release 2.3) at default setting was 
used to assemble clean reads to individual transcripts. All unique sequences with an average length of $>100 \mathrm{bp}$ were used for oligonucleotide microarray design.

The seed transcriptome has been assessed using the BUSCO 4.0.2 [95] software package by identifying core eudicot genes (eudicots_odb10) in the dataset. Twentythree of the identified core genes have been selected and aligned to homologous sequences from Jatropha curcas, Hevea brasiliensis, Manihot esculenta, Ricinus communis, Populus trichocarpa and Vitis vinifera using clustalw [96]. Phylogenetic analysis has been carried out using Beast v. 2.6 [97].

\section{GO annotation of whole seed transcripts}

Blast2GO was used to obtain the GO information. The initial blast search was carried out using BLASTX (maximum e-value of $1 \mathrm{e}-6$, gap open penalty 11 , gap extension penalty 1). Maximum 20 blast hits were retained per contig, blast hits have been submitted to the blast2go annotation database for further analysis. Furthermore, the functional annotation was used to refine annotation, and specific GO terms were labeled with their putative Biological Process (BP), Molecular Function (MF), and Cellular Component (CC). Furthermore, GO IDs were used to assign enzyme commission (EC) numbers and Kyoto Encyclopedia of Genes and Genomes (KEGG) pathways [98] to contigs.

\section{Manual annotation of specific functions}

The obtained sequences were annotated using the pipeline version of Blast2Go v2.5.0 [29]. Additional information was added to the annotation database from an InterProScan v5RC6 analysis of the sequences [99]. For protein-based similarity search, a protein sequence database of the reads was set up. Amino acid sequences for each read were defined as the most extended open reading frame of the sequence.

Specific homology searches were carried out for three distinct molecular functions of special interest: transcription factors, transporters and resistance gene analogues.

To identify transcription factors, DNA binding domain alignments were obtained from the Plant Transcription Factor (TF) Database [100]. Hidden Markov Models (HMMs) were built based on the alignment, and sequence reads were searched for these DNA binding domain models using HMMER3 [101].

Transporters were predicted based on sequence homology search using BLAST [102] against sequence entries for the Transporter Classification (TC) Database [103]. Sequence hits with an E-value lower than 1e-100 were considered as transporters of the respective class.

Reference R-gene sequences (112 genes) were acquired from the plant resistance genes (PRG) database [104] and
InterPro (IPR) domains were identified for the reference sequences with InterProScan. To predict resistance gene analogue (RGA) sequences, Blast2GO and InterProScan annotation tables were filtered for these IPR domains

Further genes or functions of interest were analyzed using text-based searches (curcins, storage proteins) in the Blast2Go/InterProScan annotation or based on the enzyme codes also included in a Blast2Go annotation table.

\section{Microarray oligonucleotide probe design}

The probes were designed (Genotypic Technology LTD.) for an $8 \times 60 \mathrm{~K}$ oligonucleotide gene expression microarray (Agilent Technologies) using all unique sequence of whole seed transcript (contigs) from the transcriptome of different maturation stages, using the Agilent's eArray software (https://earray.chem.agilent.com/earray/ ). The probes were designed in sense and antisense direction with an average probe spacing of $250 \mathrm{bp}$ (500 bp sense $+500 \mathrm{bp}$ antisense). A set of unique sequences was established as a database, and the probes were designed by tiling the contig sequences against the database. Probes specific to each transcript were selected for cross-hybridization when showing a hit with at least 30 bp and $>84 \%$ identity. Best probes were considered those showing single hits in the BLAST results.

\section{Probe labeling, hybridization, and detection}

RNA labeling, hybridization onto Agilent 8x60K oligonucleotide microarrays as well as scanning and raw data analysis was carried out according to the One-Color Microarray-Based Gene Expression Analysis Protocol provided by Agilent Technologies. Total RNA (200 ng) from each sample was used to synthesize cyanine-3 labeled cRNA using the QuickAmp Labeling kit, one Color and RNA Spike-In kit one Color (Agilent Technologies). The cyanine labeled cRNA was transcribed and purified by a T7 polymerase and RNeasy mini kits (Qiagen), respectively. Samples labeled with Cy3 (825 ng) were hybridized for $17 \mathrm{~h}$ at $65^{\circ} \mathrm{C}$ and $10 \mathrm{rpm}$ in the hybridization oven using the Gene Expression Hybridization kit (Agilent Technologies). The arrays were washed according to supplier's instructions and scanned on an Agilent G2505C scanner at $3 \mu \mathrm{m}$ resolution. Data were acquired using Agilent Feature Extraction software version 10.5.1.1. The microarrays were hybridized with probes of six stages of seeds maturation, each with three to four biological replicates.

\section{Microarray data analyses}

The R statistical (http://www.R-project.org) and Bioconductor software [105] were used to perform the preprocessing analyses of Agilent $8 \times 60 \mathrm{~K}$ oligonucleotide gene expression microarrays data. Fluorescence signal intensities from each spot were quantified. Background 
correction was performed using Agilent spatial detrending background estimate, followed by averaging of replicate spots, $\log _{2}$-transformation, KNN (K nearest neighbor) imputation of missing values and quantile normalization. The linear modeling functions of the LIMMA package were used for inference statistics [106]. Statistical significance was determined by $t$-statistic for seeds and corresponding $P$-values. Genes with Benjamini-Hochberg false discovery rate (FDR) corrected $P$-value $<1$ e- 8 were considered as significantly differentially expressed in different stages of seed maturation and leaf samples. Clustering was performed using normalized and filtered data. The differentially expressed sequences (DESs) were clustered according to their expression patterns across seed maturation stages. Normal mixture modeling for modelbased clustering (expectation-maximization) was performed with $P$-value $<1 \mathrm{e}-8$ [107]. The obtained microarray data in this study were stored in the Gene Expression Omnibus (GEO) (GSE109931).

\section{GO set enrichment analyses}

Gene set enrichment analyses were carried out according to the GO/KEGG terms using the Bioconductor GOstats package [25]. Since Jatropha is not a supported model organism, the complete GO/KEGG categories for the differentially expressed genes were identified, using the Blast2GO annotation file of the whole seed transcriptome. After building the gene-set collection, the corresponding parameter object was created followed by hyper-geometric testing. The differentially expressed genes of different maturation stages of seeds or clusters were analyzed for both over- and under-representation of GO terms, where KEGG and each GO category (BP, $\mathrm{CC}$, and MF) were analyzed separately.

Results of the GOstats analyses were plotted as flipped bar charts displaying each identified term using the negative $\log 10 P$-value for the top 15 terms. Both overand under-represented GO terms were combined in one graph, showing the $P$-value of the over-represented term on the right side and the under-represented term on the left side. In addition, heatmaps were created using the negative $\log 10 P$-value.

\section{Co-expression network analysis}

Co-expression networks based on partial correlations were calculated using the DESs with a $P$-value $<1$ e- 8 (f-statistics of the model) as described above [26, 27]. Co-expression network was constructed from the 300 most significant edges. Nodes were colored according to the cluster membership from cluster analyses of DESs, and the number of connections for the top 20 nodes with the highest number of connections to other nodes was constructed as bar plots.
Further, the genes that were expected to be involved in seed development, seed storage, and hormone crosstalking were extracted by searching the available GO annotations of each contig. A partial correlation network was constructed, and a network of the top 50 most significant edges was extracted.

\section{Quantitative real-time (qRT)-PCR using BioMark}

Primers for selected contigs from the microarray and housekeeping genes were designed using Primer3 software [28]. cDNA synthesis was performed on a total of 48 samples, including 42 test samples, four standard control samples, and two nuclease-free water (negative control) samples. For standard control, a reference sample was prepared, consisting of an equivalent pool of all test samples. In each $20 \mu \mathrm{l}$ reaction $100 \mathrm{ng}$ of total RNA per test sample as well as 800, 200, 50 and $12.5 \mathrm{ng}$ of reference RNA sample (as standard control) and only water in the two negative control samples were reverse transcribed, using the SuperScript III First-Strand Synthesis System Kit (Invitrogen) according to the manufacturer's protocol. The RT reactions were diluted 1:3, and $1.25 \mu \mathrm{l}$ of each dilution was applied to 4 different $5 \mu \mathrm{l}$ preamplification reactions, each containing $1 \mathrm{x}$ Qiagen PCR buffer, $0.8 \mathrm{mM}$ of dNTPs, $0.25 \mu \mathrm{l}$ of DMSO, 0.15 Unit of HotStarTaq DNA polymerase (Qiagen) and a pool of 48 different primer pairs (200 $\mathrm{nM}$ each). Cycling conditions for pre-amplification were $15 \mathrm{~min}$ at $95^{\circ} \mathrm{C}$ and 14 cycles of $40 \mathrm{~s}$ at $95^{\circ} \mathrm{C}, 40 \mathrm{~s}$ at $60{ }^{\circ} \mathrm{C}$ and $80 \mathrm{~s}$ at $72^{\circ} \mathrm{C}$. The cycle ended with a final step of $7 \mathrm{~min}$ at $72^{\circ} \mathrm{C}$. After preamplification, products were diluted 1:5 in nuclease-free water. QPCR amplification was performed using the BioMark system (Fluidigm) and 48.48 Dynamic Arrays. For each qPCR run $6 \mu \mathrm{l}$ sample mix were prepared, consisting of 1x Qiagen PCR Buffer (including $1.5 \mathrm{mM} \mathrm{MgCl}_{2}$ ), $0.4 \mathrm{mM} \mathrm{MgCl}_{2}, 0.96 \mathrm{mM}$ dNTPs, $0.3 \mu \mathrm{l}$ DMSO, 1x EvaGreen Binding dye, 0.18 units HotStarTaq Polymerase (QIAGEN HotStarTaq ${ }^{\mathrm{Ts}}$ PCR), $0.004 \mu \mathrm{l}$ ROX, 1x DNA binding dye (Fluidigm) and $1.5 \mu \mathrm{l}$ of pre-amplified and 1 : 5 diluted samples. In parallel, six $\mu \mathrm{l}$ of assay mix were prepared, including three $\mu \mathrm{l}$ of $2 \mathrm{x}$ Assay Loading reagent (Fluidigm), $0.3 \mu \mathrm{l}$ nuclease free water and $2.7 \mu \mathrm{l}$ of 200 $\mathrm{nM}$ primer pair pool used for pre-amplification of test samples. 48.48 Dynamic Arrays were primed, sample mix as well as assay mix were loaded with the integrated fluidic circuit (IFC) controller MX (Fluidigm) and qPCR was performed using the BioMark system (Fluidigm) according to the manufacturer's instructions. Cycling conditions were $15 \mathrm{~min}$ at $95^{\circ} \mathrm{C}$ and 40 cycles of $40 \mathrm{~s}$ at $95^{\circ} \mathrm{C}, 40 \mathrm{~s}$ at $60^{\circ} \mathrm{C}$ and $80 \mathrm{~s}$ at $72^{\circ} \mathrm{C}$. A final step of 7 min at $72^{\circ} \mathrm{C}$ ended the cycle. $\mathrm{Ct}$ values were calculated using the Fluidigm Real-Time PCR Analysis Software 4.1.2. Similar to microarray data analyses, $\mathrm{qPCR}$ data 
were normalized using quantile normalization, and linear models were calculated using the LIMMA package.

\section{Supplementary information}

Supplementary information accompanies this paper at https://doi.org/10. 1186/s12864-020-6666-1.

Additional file 1: Figure S1. Phylogenetic relationship of the seed specific transcriptome in the Euphorbiaceae family based on the sequences selected 23 core genes. Hbr: Hevea brasiliensis (rubber tree), Mes: Manihot esculenta (cassava), Jcu_own: Jatropha curcas sequences identified in this study, Jcu_ref: J. curcas NCBI reference sequences, Rco: Ricinus communis, Ptr. Populus trichocarpa, Vvi: Vitis vinifera.

Additional file 2: Figure S2. GO annotation classification of whole seed transcript sequencing data. Results are summarized for three main $\mathrm{GO}$ categories (BP, MF, and CC). The $\mathrm{x}$-axis indicates the most abundant $\mathrm{GO}$ terms, and $y$-axis represents the number of each $\mathrm{GO}$ term.

Additional file 3: Figure S3. PCA analysis explains the variance in gene expression of the six different seed maturation stages (I-VI) with their biological replications.

Additional file 4: Figure S4. The expression profiles of each microarray probe during seed developmental stages. The $x$-axis represents different developmental stages, and the $y$-axis represents the $\log _{2}$ intensity values.

Additional file 5: Figure S5. TreeMap view in REVIGO of the enriched $\mathrm{BP}$ of the six different seed maturation stages of all DESs. All terms are adjusted $P$-value cutoff at 0.05 from the enrichment analysis.

Additional file 6: Figure S6. Plot of the top 15 significantly enriched categories of KEGG pathways in the 10 identified clusters (Fig. 2). The turquoise bars show over-represented pathways and the magenta bars show under-represented pathways. The $x$-axis indicates the statistical significance of the enrichment.

Additional file 7: Figure S7. Overview of significantly enriched and over-represented pathways and enzymes related to lipid metabolism identified in different clusters. Figures generated by the pathview package to paint the gene of interests into KEGG pathways.

Additional file 8: Figure S8. Overview of significantly enriched and over-represented phenylpropanoid biosynthesis biosynthesis pathways and related enzymes identified in different clusters. Figures generated pathview package to paint the gene of interests into KEGG pathways.

Additional file 9: Figure S9. Overview of significantly enriched and over-represented flavonoid, flavone and flavonol biosynthesis and isoflavonoid biosynthesis pathways and related enzymes identified in different clusters. Figures generated by the pathview package to paint the gene of interests into KEGG pathways.

Additional file 10: Figure S10. Correlation between microarray and qRT-PCR of some DESs during seed development. The $y$-axis represented the $\log _{2}$ intensity values from microarray analysis and the $x$-axis represented the $\mathrm{Ct}$ values from the $\mathrm{qPCR}$ analysis.

Additional file 11: Table S1. Sequence comparison between obtained contigs and the Jatropha transcriptomic sequences of Kazusa DNA Research Institute (JAT_r4.5, ftp://ftp.kazusa.or.jp/pub/Jatropha/), as well as Chinese Academy of Sciences (JatCur_1.0, ftp://ftp.ncbi.nih.gov/ genomes/Jatropha_curcas/).

Additional file 12: Table S2. GO classification of annotated transcripts of whole seed transcript sequencing data. Results are summarized for three main $\mathrm{GO}$ categories (BP, MF, and CC).

Additional file 13: Table S3. Annotation of whole transcript sequencing data according to Blast2Go, InterProScan, and KEGG. Manual annotation was carried out to predict RGA, TF and transporter sequences using the Plant Resistance Genes, the Plant Transcription Factor, and the Transporter Classification Databases.

Additional file 14: Table S4 Mapping of the identified enzymes and their corresponding contigs to KEGG pathways.
Additional file 15: Table S5. Relative transcript expression value $\left(\log _{2}\right)$ of all DESs (with a cut-off of $P$-value $<1$ e-8) in each developmental stages and related clusters.

Additional file 16: Table S6. Pairwise comparison of transcripts expression values $\left(\log _{2}\right)$ between different maturation stages (with a cutoff of $P$-value $<1 \mathrm{e}-8$ ).

Additional file 17: Table S7. The number of over- and underrepresented $\mathrm{GO}$ terms of each category (BP, MF and CC), and related contigs in each cluster.

Additional file 18: Table S8. GO and KEGG enrichment analysis of significantly over- and under-represented GO terms of each category (BP, MF, and (C) and KEGG pathways for each cluster.

Additional file 19: Table S9. The enriched pathways of all significantly DESs for each cluster.

Additional file 20: Table S10. The list of 75 selected transcripts from the DESs represented in seeds and three housekeeping genes, which were used for qRT-PCR.

Additional file 21: Table S11. Relative expression value of all selected transcript from DESs represented in seeds and three housekeeping genes, which were used for qRT-PCR.

\section{Abbreviations}

GO: Gene ontology; DESs: Differentially expressed sequences; CO2: Carbon dioxide; J. curcas: Jatropha curcas; TF: Transcription factor; HMMs: Hidden Markov Models; IPR: InterPro; RGA: Resistance gene analogue; BP: Biological process; MF: Molecular function; CC: Cellular component; EC: Enzyme commission; KEGG: Kyoto encyclopedia of genes and genomes; KNN: K nearest neighbor; FDR: False discovery rate; GEO: Gene expression omnibus; HQ: High quality; Xhyb: Cross-hybridizing probes; PCA: Principle components analysis; S.D.: Standard deviation; CDR1: Aspartic proteinase; DFR: Dihydroflavonol reductase; TT8: Transparent Testa 8; ER: Endoplasmic reticulum; CAC2: Carboxylase biotin carboxylase subunit; KASII: Beta-ketoacylacp synthase II; MOD1: Mosaic death 1; FAB2: Fatty acid biosynthesis 2; FatA: Fata acyl-acp thioesterase; FAE1: Fatty acid elongation1; FAD2: Fatty acid desaturase2; LEC1: Leafy cotyledon1; DGAT1: Diacylglycerol acyltransferase 1; FUS3: FUSCA3; CDS2: Cytidinediphosphate diacylglycerol synthase 2; ACC: Acetyl-CoA carboxylase; MCMT: Malonyl-CoA acyl carrier protein (ACP) transacylase; KAS III: Beta-ketoacyl-ACP synthase III; KAR: 3oxoacyl-ACP reductase; KASI: Beta-ketoacyl-ACP synthase I; KASII: Betaketoacyl-ACP synthase II; AAD: Acyl-ACP desaturase; OAH: Oleoyl-ACP hydrolase; FAT: Acyl-ACP thioesterase; DAG: Diacylglycerol; TAG: Triacylglycerol; PDAT1: Phospholipid: diacylglycerol acyltransferase 1; G3P: Glycerol-3-phosphate; GPAT: G3P acyltransferase; LPA: Lysophosphatidic acid; PA: Phosphatidic acid; LPAAT: LPA acyltransferase; PC: Phosphatidylcholine; DGAT: Diacylglycerol O-acyltransferase; PDAT: Phospholipid diacylglycerol acyltransferase; ATP: Diacylglycerol kinase; NAD: Aldehyde dehydrogenase; PAL: Phenylalanine ammonia-lyase; 4CL: 4Coumarate: CoA ligase; COMT: Caffeic acid 3-O-methyltransferase; CCOAOMT: Caffeoyl-CoA O-methyltransferase; CCR: Cinnamoyl-CoA reductase; ACC: Aminocyclopropanecarboxylate

\section{Acknowledgements}

We would like to thank Debesaye Senbeto (Agricultural Research Center Adama, Melkasa, Ethiopia) and Yoseph Tewodros for their help in providing Jatropha samples.

\section{Authors' contributions}

FM and ML designed and conceived the project. FM carried out all experiments, participated in data analyses and wrote the final manuscript. TD, KV, SP, HT participated in data analyses and manuscript editing. All authors read and approved the final manuscript.

\section{Funding}

This research was financially supported by the Austrian Science Fund (FWF, P 23836). Authors thank the Austrian Research Promotion Agency (FFG), Bioplant R\&D, Vienna, for the financial support. The support of the Ministry for Innovation and Technology within the framework of the Higher Education Institutional Excellence Program (NKFIH-1159-6/2019) in the scope 
of plant breeding and plant protection researches of Szent István University is acknowledged. The funding bodies played no role in the design of the study and collection, analysis, and interpretation of data and in writing the manuscript.

\section{Availability of data and materials}

The whole dataset generated and analyzed during the current study is available from the corresponding authors on request. Raw sequence reads are accessible in the NCBI SRA database (SRR7701127), assembled transcripts have been uploaded to TSA under the accession GIKD00000000. The version described in this paper is the first version, GIKD01000000.

\section{Ethics approval and consent to participate}

Not applicable.

\section{Consent for publication}

Not applicable

\section{Competing interests}

The authors declare that they have no competing interests.

\section{Author details}

${ }^{1}$ Plant Functional Genomics, Department of Biotechnology, BOKU-VIBT, University of Natural Resources and Life Sciences, Muthgasse 18, 1190 Vienna, Austria. ${ }^{2}$ Department of Viticulture, Szent István University, Villányi út 29-43, 1118 Budapest, Hungary. ${ }^{3}$ Center for Health and Bioresources, Molecular Diagnostics, Austrian Institute of Technology (AIT), Giefinggasse 4, 1210 Vienna, Austria. ${ }^{4}$ Austrian Center of Biological Resources (ACBR), Department of Biotechnology, BOKU-VIBT, University of Natural Resources and Life Sciences, Muthgasse 18, 1190 Vienna, Austria. ${ }^{5}$ Plant Biotechnology Unit, Department of Biotechnology, BOKU-VIBT, University of Natural Resources and Life Sciences, Muthgasse 18, 1190 Vienna, Austria.

Received: 1 August 2019 Accepted: 11 March 2020

Published online: 09 April 2020

\section{References}

1. Ovando-Medina I, Espinosa-García F, Núñez-Farfán J, Salvador-Figueroa M. Does biodiesel from Jatropha curcas represent a sustainable alternative energy source? Sustainability. 2009;1:1035-41.

2. Blesgraaf RAR. Water use of Jatropha, Hydrological impacts of Jatropha curcas L. MSC Thesis. Delft: Delft University of Technology; 2009.

3. Grass M. Jatropha curcas L: visions and realities. J Agric Rural Dev Trop Subtrop. 2009:110:29-38.

4. Rajaona AM, Sutterer N, Asch F. Potential of waste water use for Jatropha cultivation in arid environments. Agriculture. 2012;2:376-92.

5. Maghuly F, Laimer M. Jatropha curcas, a biofuel crop: functional genomics for understanding metabolic pathways and genetic improvement. Biotechnol J. 2013;8:1172-82.

6. Chhetri AB, Tango MS, Budge SM, Watts KC, Islam MR. Non-Edible Plant Oils as New Sources for Biodiesel Production. Int J Mol Sci. 2008;9:169-80.

7. Rao PV, Rao GS. Production and characterization of Jatropha oil methyl ester international. J of Eng Res. 2013:2:141-5.

8. Agbogidi OM, Akparobi SO, Eruotor PG. Health and environmental benefits of Jatropha curcas Linn. App Sci Re. 2013;1:36-9.

9. Warra AA. Cosmetic potentials of physic nut (Jatropha curcas Linn) seed oil: a review. Am J Sci Ind Res. 2012:3:358-66.

10. Bayen P, Sop T K, Lykke A M, Thiombiano A. Does Jatropha curcas L show resistance to drought in the Sahelian zone of West Africa? A case study from Burkina Faso Solid Earth. Solid Earth. 2015;6:525-31.

11. Achten W, Nielsen L, Aerts R, Lengkeek A, Kjær ED, Trabucco A, Hansen JK, Maes WH, Graudal L, Akinnifesi FK, Muys B. Towards domestication of Jatropha curcas. Biofuel. 2010;1:91-107.

12. Sabandar CW, Ahmat N, Jaafar FM, Sahidin I. Medicinal property, phytochemistry and pharmacology of several Jatropha species (Euphorbiaceae): A review. Phytochemistry. 2013;85:7-29.

13. Sabandar CW, Ahmat N, Jaafar FM, Sahidin I. Medicinal property, phytochemistry and pharmacology of several Jatropha species (Euphorbiaceae): a review. Phytochemistry. 2013;85:7-29.

14. Ha J, Shim S, Lee T, Kang YJ, Hwang WJ, Jeong H, Laosatit K, Lee J, Kim SK, Satyawan D, Lestari P, Yoon MY, Kim MY, Chitikineni A, Tanya P, Somta P,
Srinives P, Varshney RK, Lee S. Genome sequence of Jatropha curcas L., a non-edible biodiesel plant, provides a resource to improve seed-related traits. Plant Biotechnol J. 2019;17:517-30.

15. Jiang H, Wu P, Zhang S, Song C, Chen Y, Li M, Jia Y, Fang X, Chen F, Wu G. Global analysis of gene expression profiles in developing physic nut (Jatropha curcas L) seeds. PLoS One. 2012;7:e36522.

16. Costa G, Del Bem CK, Lima L, Cunha A, et al. Transcriptome analysis of the oil-rich seed of the bioenergy crop Jatropha curcas L. BMC Genomics. 2010; 11:462.

17. King AJ, Li Y, Graham IA. Profiling the developing Jatropha curcas $L$ seed transcriptome by pyrosequencing. Bioenerg Res. 2011;4:211-21.

18. King A J, Montes L R, Clarke J G, Affleck J, Li Y Witsenboer, H van der Vossen, E van der Linde, P Tripathi, Y Tavares, E Shukla, P Rajasekaran, T van Loo, EN Graham I A Linkage mapping in the oilseed crop Jatropha curcas L reveals a locus controlling the biosynthesis of phorbol esters which cause seed toxicity Plant Biotechnol J, 2013; 11: 986-996.

19. Hirakawa H, Tsuchimoto S, Sakai H, Nakayama S, Fujishiro T, Kishida Y, Kohara M, Watanabe A, Yamada M, Aizu T, Toyoda A, Fujiyama A, Tabata S, Fukui K, Sato S. Upgraded genomic information of Jatropha curcas L. Plant Biotechnol. 2012;29:123-30.

20. Sato S, Hirakawa H, Isobe S, Fukai E, Watanabe A, et al. Sequence analysis of the genome of an oil-bearing tree, Jatropha curcas L. DNA Res. 2011;18:65-76.

21. Zhang L, Zhang C, Wu P, Chen Y, Li M, Jiang H, Wu G. Global analysis of gene expression profiles in physic nut (Jatropha curcas L) seedlings exposed to salt stress. PLoS One 2014:9:e97878.

22. Wu P, Zhou C, Cheng S, Wu Z, Lu W, Han J, et al. Integrated genome sequence and linkage map of physic nut (Jatropha curcas L), a biodiesel plant. Plant J. 2015;81:810-21.

23. Qu J, Mao HZ, Chen W, Gao SQ, et al. Development of marker-free transgenic Jatropha plants with increased levels of seed oleic acid. Biotechnol Biofuels. 2012;5:10.

24. Ye J, Wang C, Sun Y, Qu J, Mao H, Chua N-H. Overexpression of a transcription factor increases lipid content in a Woody Perennial Jatropha curcas. Front Plant Sci. 2018;9:1479.

25. Falcon S, Gentleman R. Using GOstats to test gene lists for GO term association. Bioinformatics. 2007;23:257-8.

26. Schaefer J, Opgen-Rhein R, Strimmer K. GeneNet. Modeling and inferring gene networks R package version 1.2.13. 2015; https://CRAN.R-project.org/ package $=$ GeneNet.

27. Opgen-Rhein R, Strimmer K. From correlation to causation networks: a simple approximate learning algorithm and its application to highdimensional plant gene expression data. BMC Syst Biol. 2007:1:37.

28. Rozen S, Skaletsky H. Primer3 on the WWW for general users and for biologist programmers. In: Misener S, Krawetz S, editors. Bioinformatics Methods and Protocols, vol. 132. Totwa: Humana Press. 1999. p. 36586.

29. Conesa A, Götz S, García-Gómez JM, Terol J, Talón M, Robles M. Blast2GO: a universal tool for annotation, visualization, and analysis in functional genomics research. Bioinformatics. 2005;21:3674-6.

30. Altschul SF, Madden TL, Schäffer A, Zhang J, Miller W, Lipman DJ. Gapped BLAST and PSI-BLAST: a new generation of protein database search programs. Nucleic Acids Res. 1997;25:3389-402.

31. Afzal AJ, Wood AJ, Lightfoot DA. Plant receptor-like serine threonine kinases. Roles in signalling and plant defense. Molecular plant-microbe interactions. MPMI. 2008;21:507-17.

32. Sekhwal MK, Li P, Lam I, Wang X, Cloutier S, You FM. Disease resistance gene analogs (RGAs) in plants. Int J Mol Sci. 2015;16:19248-90.

33. Baud S, Boutin J-P, Miquel M, Lepiniec L, Rocha C. Integrated overview of seed development in Arabidopsis thaliana ecotype. Plant Physiol Biochem. 2002:40:151-60

34. Jolivet P, Boulard C, Bellamy A, Valot B, d'Andréa S, Zivy M, Nesi N, Chardot T. Oil body proteins sequentially accumulate throughout seed development in Brasica napus. J Plant Physiol. 2011;168:2015-20.

35. Righetti KV, Pelletier JS, et al. Inference of longevity-related genes from a robust co-expression network of seed maturation identifies regulators linking seed storability to biotic defense-related pathways. Plant Cell. 2015; 27:2692-708.

36. Leubner-Metzger G. Functions and regulation of b-1,3-glucanases during seed germination, dormancy release and after-ripening. Seed Sci Res. 2003; 13:17-34. 
37. Shah M, Soares EL, Carvalho PC, Soares AA, Domont GB, Nogueira FC, Campos FA. Proteomic analysis of the endosperm ontogeny of Jatropha curcas L. seeds. J Proteome Res. 2015;14:2557-68.

38. Liu H, Wang C, Komatsu S, He M, Liu G, Shen S. Proteomic analysis of the seed development in Jatropha curcas: from carbon flux to the lipid accumulation. J Proteome. 2013:91:23-40.

39. Borisjuk L, Nguyen TH, Neuberger T, Rutten T, Tschiersch H, et al. Gradients of lipid storage, photosynthesis and plastid differentiation in developing soybean seeds. New Phytologist. 2005;167:761-76.

40. Whetten R, Sederoff R. Lignin biosynthesis. Plant Cell. 1995;7:1001-13.

41. Li H, Dong Y, Yang J, Liu X, Wang Y, Yao N, Guan L, Wang N, Wu J, Li X. De novo transcriptome of safflower and the identification of putative genes for oleosin and the biosynthesis of flavonoids. PLoS One. 2012;7:e30987.

42. Qu C, Zhao H, Fu F, Wang Z, Zhang K, Zhou Y, Wang X, Wang R, Xu X, Tang Z, Lu K, Zhang K, Zhou Y, Wang X, Wang R, XU X, Tang Z, Lu K. GenomeWide Survey of Flavonoid Biosynthesis Genes and Gene Expression Analysis between Black- and Yellow-Seeded Brassica napus. Front Plant Sci. 2016;7: 1755.

43. Nazarov PV, Muller A, Kaoma T. Nicot N, Maximo C, Birembaut P, Tran NL, Dittmar G, Vallar L. RNA sequencing and transcriptome arrays analyses show opposing results for alternative splicing in patient derived samples. BMC Genomics. 2017;18:443

44. Gou M, Yang X, Zhao Y, Ran X, Song Y, Liu CJ. Cytochrome b5 is an obligate Electron shuttle protein for Syringyl lignin biosynthesis in Arabidopsis. Plant Cell. 2019;31:1344-66.

45. Hwang YT, Pelitire SM, Henderson MP, Andrews DW, Dyer JM, Mullen RT. Novel targeting signals mediate the sorting of different isoforms of the tailanchored membrane protein cytochrome b5 to either endoplasmic reticulum or mitochondria. Plant Cell. 2004;16:3002-19.

46. Smith MA, Jonsson L, Stymne S, Stobart K. Evidence for cytochrome b5 as an electron donor in ricinoleic acid biosynthesis in microsomal preparations from developing castor bean (Ricinus communis L). Biochem J. 1992;287:141-4.

47. Bafor M, Smith MA, Jonsson L, Stobart K, Stymne S. Biosynthesis of vernoleate (cis-12-epoxyoctadeca-cis-9-enoate) in microsomal preparations from developing endosperm of Euphorbia lagascae arch. Biochem Biophys. 1993;303:145-51.

48. Napier JA, Michaelson LV, Sayanova O. The role of cytochrome b5 fusion desaturases in the synthesis of polyunsaturated fatty acids. Prostaglandins Leukot Essent Fatty Acids. 2003;68:135-43.

49. Sharma N, Anderson M, Kumar A, Zhang Y, Giblin EM, Abrams SR, Zaharia LI, Taylor DC, Fobert PR. Transgenic increases in seed oil content are associated with the differential expression of novel Brassica-specific transcripts. BMC Genomics. 2008:9:619.

50. Chen M, Xuan L, Wang Z, Zhou L, Li Z, Du X, Ali E, Zhang G, Jiang L. TRAN SPARENT TESTA8 inhibits seed fatty acid accumulation by targeting several seed development regulators in Arabidopsis. Plant Physiol. 2014;165:905-16.

51. Akhov L, Ashe P, Tan Y, Datla R, Selvaraj G. Proanthocyanidin biosynthesis in the seed coat of yellow-seeded, canola quality Brassica napus YN01-429 is constrained at the committed step catalyzed by dihydroflavonol 4reductase. Botany. 2009;87:616-25.

52. Carvalho Lemos V, Reimer JJ, Wormit A. Color for life: biosynthesis and distribution of phenolic compounds in pepper (Capsicum annuum). Agriculture. 2019;9:81

53. Guo WJ, Ho TH. An abscisic acid-induced protein, HVA22, inhibits gibberellin-mediated programmed cell death in cereal aleurone cells. Plant Physiol. 2008;147:1710-22.

54. Kotak S, Vierling E, Bäumlein H, Pv K-D. A novel transcriptional Cascade regulating expression of heat stress proteins during seed development of Arabidopsis. Plant Cell. 2007;19:182-95.

55. DeRocher A, Vierling E. Cytoplasmic HSP70 homologues of pea: differential expression in vegetative and embryonic organs. Plant Mol Biol. 1995;27:441-56.

56. Sung DY, Vierling E, Guy CL. Comprehensive expression profile analysis of the Arabidopsis Hsp70 gene family. Plant Physiol. 2001;126:789-800.

57. Wakasa Y, Yasuda H, Oono Y, Kawakatsu T, Hirose S, Takahashi H, Hayashi S, Yang L, Takaiwa F. Expression of ER quality control-related genes in response to changes in BiP1 levels in developing rice endosperm. Plant J. 2011;65:675-89.

58. Sarkar NK, Kundnani P, Grover A. Functional analysis of Hsp70 superfamily proteins of rice (Oryza sativa). Cell Stress Chaperones. 2013;18:427-37.

59. Kang J, Park J, Choi H, Burla B, Kretzschmar T, Lee Y, Martinoia E. Plant ABC Transporters. Arabidopsis Book. 2011;9: e0153.
60. Ko D, Kang J, Kiba T, Park J, Kojima M, Do J, Kim KY, Kwon M, Endler A, Song W-Y, et al. Arabidopsis ABCG14 is essential for the root-to-shoot translocation of cytokinin. Proc Natl Acad Sci USA. 2014;111:7150-5.

61. Zhang H, Zhu H, Pan Y, Yu Y, Luan S, Li L. A DTX/MATE-type transporter facilitates abscisic acid efflux and modulates ABA sensitivity and drought tolerance in Arabidopsis. Mol Plant. 2014;7:1522-32.

62. Xu C, Fan J, Froehlich JE, Awai K, Benning C. Mutation of the TGD1 chloroplast envelope protein affects phosphatidate metabolism in Arabidopsis. Plant Cell. 2005;17:3094-110.

63. Kuromori T, Miyaji T, Yabuuchi H, Shimizu H, Sugimoto E, Kamiya A, Moriyama Y, Shinozaki K. ABC transporter AtABCG25 is involved in abscisic acid transport and responses. Proc Natl Acad Sci USA. 2010;107:2361-6.

64. Hwang JU, Song WY, Hong D, Ko D, Yamaoka Y, Jang S, Yim S, Lee E, Khare $D$, Kim $K$, et al. Plant $A B C$ transporters enable many unique aspects of a terrestrial plant's lifestyle. Mol Plant. 2016;9:338-55.

65. Hofvander P, Ischebeck T, Turesson H, Kushwaha SK, Feussner I, Carlsson AS, Andersson M. Potato tuber expression of Arabidopsis WRINKLED1 increase triacylglycerol and membrane lipids while affecting central carbohydrate metabolism. Plant Biotechnol J. 2016;14:1883.

66. Chia TYP, Pike MJ, Rawsthorne S. Storage oil breakdown during embryo development of Brassica napus (L.). J Exp Bot. 2005;56:1285.

67. Li H, Peng Z, Yang X, Wang W, Fu J, Wang J, Han Y, Chai Y, Guo T, Yang N, Liu J, Warburton ML, Cheng Y, Hao X, Zhang P, Zhao J, Liu Y, Wang G, Li J, Yan J. Genome-wide association study dissects the genetic architecture of oil biosynthesis in maize kernels. Nat Genet. 2013;45:43-50.

68. Cernac A, Benning C. WRINKLED1 encodes an AP2/EREB domain protein involved in the control of storage compound biosynthesis in Arabidopsis. Plant J. 2004;40:575-85.

69. Deng S, Mai Y, Shui L, Niu J. WRINKLED1 transcription factor orchestrates the regulation of carbon partitioning for C18:1 (oleic acid) accumulation in Siberian apricot kernel. Sci Rep. 2019;9:2693.

70. Maeo K, Tokuda T, Ayame A, Mitsui N, Kawai T, Tsukagoshi H, Ishiguro S, Nakamura K. An AP2-type transcription factor, WRINKLED1, of Arabidopsis thaliana binds to the AW-box sequence conserved among proximal upstream regions of genes involved in fatty acid synthesis. Plant J. 2010;60:476-87.

71. Shi L, Katavic V, Yu Y, Kunst L, Haughn G. Arabidopsis glabra2 mutant seeds deficient in mucilage biosynthesis produce more oil. Plant J. 2012;69:37-46.

72. Harma A, Shahzad B, Rehman A, Bhardwaj R, Landi M, Zheng B. Response of Phenylpropanoid pathway and the role of polyphenols in plants under abiotic stress. Molecules. 2019;24:2452.

73. Min T, Bao Y, Zhou B, Yi Y, Wang L, Hou W, Ai Y, Wang H. Transcription profiles reveal the regulatory synthesis of phenols during the development of Lotus rhizome (Nelumbo nucifera Gaertn). Int J Mol Sci. 2019:20:2735.

74. Xu R, Wang R, Liu A. Expression profiles of genes involved in fatty acid and triacylglycerol synthesis in developing seeds of Jatropha (Jatropha curcas L). Biomass Bioenergy. 2011;35:1683-92.

75. Maisonneuve S, Bessoule J-J, Lessire R, Delseny M, Roscoe TJ. Expression of rapeseed microsomal Lysophosphatidic acid Acyltransferase Isozymes enhances seed oil content in Arabidopsis. Plant Physiol. 2010;152:670-84.

76. Chen J, Tan RK, Guo XJ, Fu ZL, Wang Z, Zhang ZY, Tan XL. Transcriptome analysis comparison of lipid biosynthesis in the leaves and developing seeds of Brassica napus. PLoS One. 2015;10:e0126250.

77. Chapman KD, Ohlrogge JB. Compartmentation of triacylglycerol accumulation in plants. J Biol Chem. 2012;287:2288-94.

78. Routaboul J-M, Benning C, Bechtold N, Caboche M, Lepiniec L. The TAG1 locus of Arabidopsis encodes for a diacylglycerol acyltransferase. Plant Physiol Biochem. 1999:37:831-40.

79. Li Y, Kim Jl, Pysh L, Chapple C. Four isoforms of Arabidopsis 4-Coumarate: CoA ligase have overlapping yet distinct roles in phenylpropanoid metabolism. Plant Physiol. 2015;169:2409-21.

80. Zhang M, Fan J, Taylor DC, Ohlrogge JB. DGAT1 and PDAT1 acyltransferases have overlapping functions in arabidopsis triacylglycerol biosynthesis and are essential for normal pollen and seed development. The Plant Cell. 2009; 2:3885-901.

81. Wang L, Yu S, Tong C, Zhao Y, Liu Y, Song C, Zhang Y, et al. Genome sequencing of the high oil crop sesame provides insight into oil biosynthesis. Genome Biol. 2014;15:R39.

82. Brown AP, Kroon JTM, Swarbreck D, Febrer M, Larson TR, Graham IA, Caccamo M, et al. Tissue-specific whole transcriptome sequencing in castor, directed at understanding triacylglycerol lipid biosynthetic pathways. PLoS One. 2012;7:e30100 
83. Basnet R, Moreno-Pachon N, Lin K, Bucher J, Visser RGF, Maliepaard C, Bonnema G. Genome-wide analysis of coordinated transcript abundance during seed development in different Brassica rapa morphotypes. BMC Genomics. 2013;14:840

84. Gu K, Yi C, Tian D, Sangha J, Hong Y, Yin Z. Expression of fatty acid and lipid biosynthetic genes in developing endosperm of Jatropha curcas. Biotechnol Biofuels. 2012;5:47

85. Chen M-S, Wang G-J, Wang R-L, et al. Analysis of expressed sequence tags from biodiesel plant Jatropha curcas embryos at different developmental stages. Plant Sci. 2011;181:696-700.

86. Chandran D, Sankararamasubramanian HM, Kumar MA, Parida A. Differential expression analysis of transcripts related to oil metabolism in maturing seeds of Jatropha curcas L. Physiol Mol Biol Plants. 2014;20:181-90.

87. Biała W, Jasiński M. The phenylpropanoid case - it is transport that matters. Front Plant Sci. 2018:9:1610.

88. Ehlting J, Büttner D, Wang Q, Douglas CJ, Somssich IE, Kombrink E. Three 4coumarate:coenzyme a ligases in Arabidopsis thaliana represent two evolutionarily divergent classes in angiosperms. Plant J. 1999;19:9-20.

89. Lulin H, Xiao Y, Pei S, Wen T, Shangqin H. The first Illumina-based de novo transcriptome sequencing and analysis of safflower flowers. PLoS One. 2012; 7:e38653.

90. Kleindt CK, Stracke R, Mehrtens F, Weisshaar B. Expression analysis of flavonoid biosynthesis genes during Arabidopsis thaliana silique and seed development with a primary focus on the proanthocyanidin biosynthetic pathway. BMC Res Notes. 2010;3:255.

91. Tohge T, Perez de Souza L, Fernie AR. Current understanding of the pathways of flavonoid biosynthesis in model and crop plants. J Exp Bot. 2017:68:4013-28

92. Fait A, Angelovici R, Less H, Ohad I, Urbanczyk-Wochniak E, Fernie AR, Galili $\mathrm{G}$. Arabidopsis seed development and germination is associated with temporally distinct metabolic switches. Plant Physiol. 2006;142:839-54.

93. Peng F, Weselake R. Gene coexpression clusters and putative regulatory elements underlying seed storage reserve accumulation in Arabidopsis. BMC Genomics. 2011;12:286.

94. Silva LJ, Dias DCFS, Milagres CC, Dias LAS. Relationship between fruit maturation stage and physiological quality of physic nut (Jatropha curcas L.) seeds. Rev Ciência e Agrotecnol. 2012;36:39-44.

95. Seppey M, Manni M, Zdobnov EM. BUSCO: assessing genome assembly and annotation completeness. In: Kollmar M, editor. Gene prediction. Methods in Molecular Biology, vol. 1962. New York: Humana; 2019. p. 227-45.

96. Larkin MA, Blackshields G, Brown NP, Chenna R, McGettigan PA, McWilliam $H$, et al. Version 2.0. Bioinformatics. 2007;23:2947-8.

97. Bouckaert R, Vaughan TG, Barido-Sottani J, Duchêne S, Fourment GA, et al. BEAST 2.5: An advanced software platform for Bayesian evolutionary analysis. PLoS Comput Biol. 2019;15:e1006650

98. Ogata H, Goto S, Sato K, Fujibuchi W, Bono H, Kanehisa M. KEGG: Kyoto encyclopedia of genes and genomes. Nucleic Acids Res. 1999;27:29-34.

99. Quevillon E, Silventoinen V, Pillai S, Harte N, Mulder N, Apweiler R, Lopez R. InterProScan: protein domains identifier. Nucleic Acids Res. 2005:33:116-20.

100. Jin J, Zhang H, Kong L, Gao G, Luo J. PlantTFDB 30: a portal for the functional and evolutionary study of plant transcription factors. Nucleic Acids Res. 2014;42:1182-7.

101. Eddy SR. Accelerated profile HMM searches. PLoS Comput Biol. 2011;7: e1002195.

102. Altshuler DM, Gibbs RA, Peltonen L, Altshuler DM, Gibbs RA, et al. Integrating common and rare genetic variation in diverse human populations. Nature. 2010;467:52-8.

103. Saier MH, Yen MR, Noto K, Tamang DG, Elkan C. The transporter classification database: recent advances. Nucleic Acids Res. 2009:37:274-8.

104. Sanseverino W, Hermoso A, D’Alessandro R, Vlasova A, Andolfo G, Frusciante L, Lowy E, Roma G, Ercolano MR. PRGdb 20: towards a community-based database model for the analysis of R-genes in plants. Nucleic Acids Res. 2013:41:1167-71.

105. R Core Team. R: A language and environment for statistical computing $R$ Foundation for Statistical Computing, Vienna, Austria. 2012. http://wwwRproject.org/.

106. Smyth G. Limma: linear models for microarray data. In: Bioinformatics and Computational Biology Solutions Using R and Bioconductor Statistics for Biology and Health (Gentleman R, Carey VJ, Huber W, Irizarry RA, Dudoit S eds). New York: Springer; 2005. p. 397-420.
107. Fraley C, Raftery AE. Model-based clustering, discriminant analysis, and density estimation. J Am Stat Assoc. 2002;97:611-31.

\section{Publisher's Note}

Springer Nature remains neutral with regard to jurisdictional claims in published maps and institutional affiliations.

\section{Ready to submit your research? Choose BMC and benefit from:}

- fast, convenient online submission

- thorough peer review by experienced researchers in your field

- rapid publication on acceptance

- support for research data, including large and complex data types

- gold Open Access which fosters wider collaboration and increased citations

- maximum visibility for your research: over $100 \mathrm{M}$ website views per year

At $\mathrm{BMC}$, research is always in progress.

Learn more biomedcentral.com/submissions 